\title{
Margin for In-Vessel Retention in the APR1400 - VESTA and SCDAP/RELAP5-3D ${ }^{\odot}$ Analyses
}

\author{
J. L. Rempe and D. L. Knudson
}

December 2004

Idaho National Engineering and Environmental Laboratory Bechtel BWXT Idaho, LLC 


\title{
Margin for In-Vessel Retention in the APR1400 - VESTA and SCDAP/RELAP5-3D ${ }^{\odot}$ Analyses
}

\author{
J. L. Rempe and D. L. Knudson
}

December 2004

Idaho National Engineering and Environmental Laboratory

Idaho Falls, Idaho 83415

Prepared for the U.S. Department of Energy Under DOE Idaho Operations Office Contract DE-AC07-99ID13727 


\section{CONTENTS}

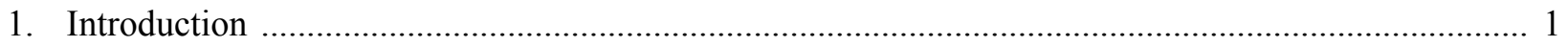

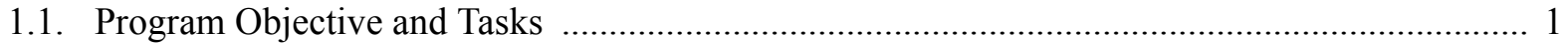

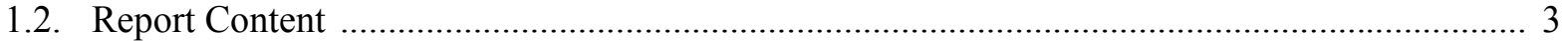

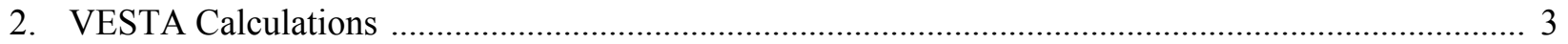

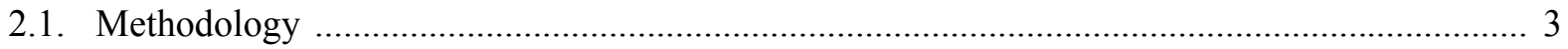

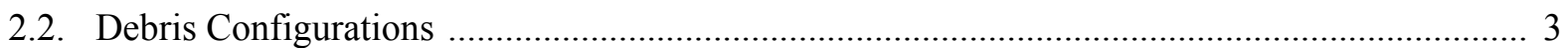

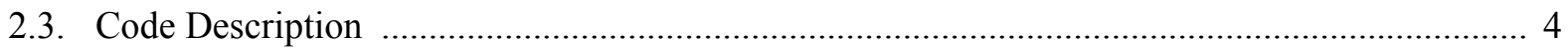

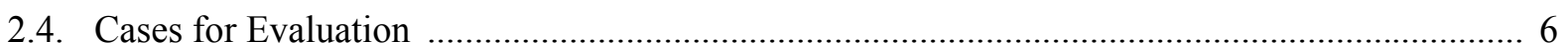

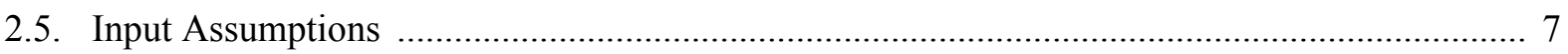

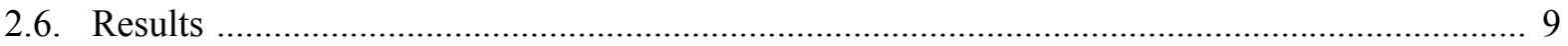

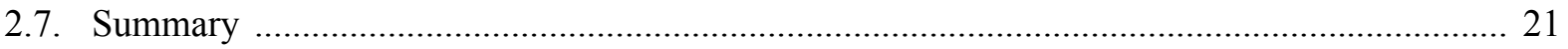

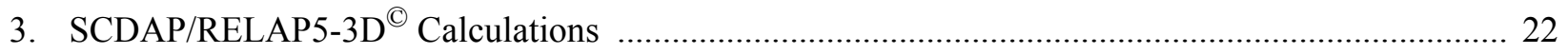

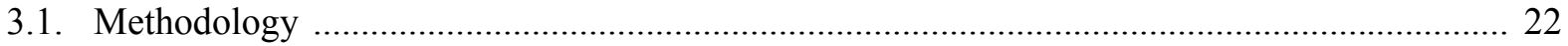

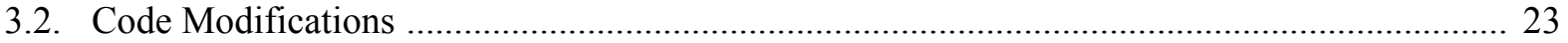

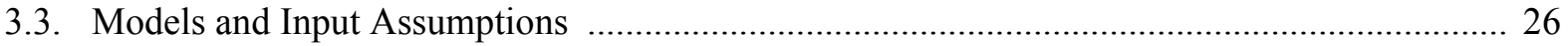

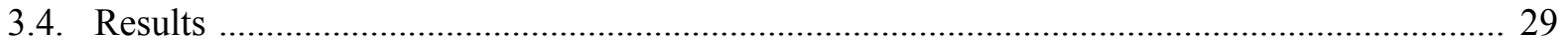

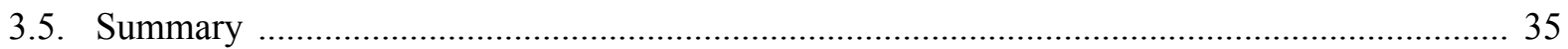

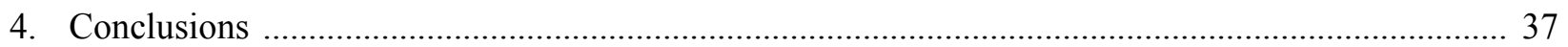

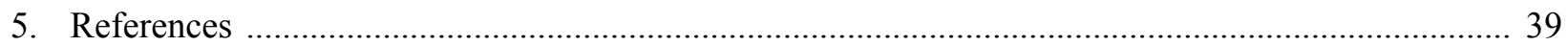




\section{FIGURES}

1. INERI applied key experimental facilities and state-of-the-art analytical tools......................... 2

2. Project organization illustrating key tasks and participating organizations................................. 2

3. Debris configurations evaluated for the APR1400 ........................................................... 4

4. CHF associated with various ERVC conditions. ............................................................... 10

5. Heat fluxes to the vessel wall for Configuration A base case ................................................. 11

6. CHF ratios for Configuration A base case ....................................................................... 11

7. CHF ratio pdfs for Configuration A base case ................................................................. 12

8. Heat fluxes assuming various metallic layer steel masses for Configuration A base case........... 13

9. CHF ratios assuming various metallic layer steel masses for Configuration A base case............ 13

10. Heat fluxes assuming various relocation time delays for Configuration A base case................. 14

11. CHF ratios assuming various relocation times delay for Configuration $\mathrm{A}$ base case................. 15

12. CHF ratios assuming various ERVC enhancements for Configuration A base case.................. 16

13. CHF ratio pdfs for Configuration A base case with vessel coating. ........................................ 16

14. CHF ratio pdfs for Configuration A base case with enhanced vessel/insulation design. ............ 17

15. CHF ratio pdfs for Configuration A base case with "combined" ERVC enhancements............. 17

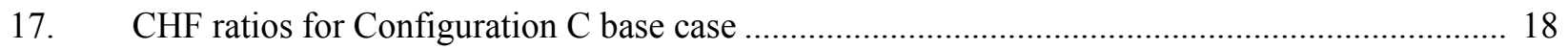

16. Heat fluxes to the vessel wall for Configuration $\mathrm{C}$ base case .................................................. 18

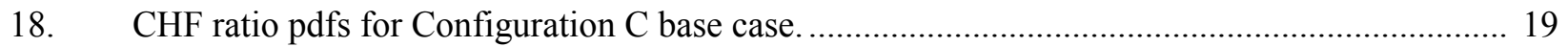

19. Heat fluxes for various metallic layer steel masses in Configuration $\mathrm{C}$ base case.....................20

20. CHF ratios for various metallic layer steel masses in Configuration $\mathrm{C}$ base case ...................... 20

21. CHF ratios assuming various IVR enhancements for Configuration $\mathrm{C}$ base case...................... 21

22. Illustration of an angular position relative to heat transfer from a hemispherical surface........... 24

23. Curves for saturated boiling at atmospheric pressure from a vessel with a microporous coating

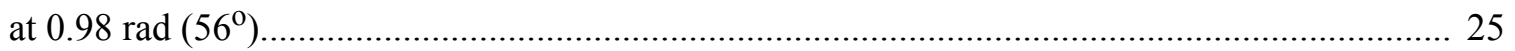

24. Simplified SCDAP/RELAP5-3D ${ }^{\complement}$ model of the APR1400 lower head thermal-hydraulics....... 26

25. COUPLE two-dimensional finite-element mesh representing the lower head of the APR1400 reactor vessel (with selected node numbers).

26. Nusselt number ratio as a function of the angle from molten pool centerline.

27. The ratio of vessel surface heat flux to the CHF for a vessel with a microporous coating at $9000 \mathrm{~s}$

28. Through-wall temperature profile (from Node 9 in Figure 25) for a vessel with a microporous coating.

29. Through-wall temperature profile (from Node 12 in Figure 25) for a vessel with a microporous coating. 
30. The ratio of vessel surface heat flux to the CHF for Node 12 on a vessel with a microporous coating (see Figure 25 for node position).

31. The ratio of vessel surface heat flux to the CHF for a vessel with a microporous coating at $6000 \mathrm{~s}$. 35

32. Through-wall temperature profile (from Node 9 in Figure 25) for a plain vessel. ..................... 36

33. Through-wall temperature profile (from Node 12 in Figure 25) for a plain vessel.................... 36 


\section{TABLES}

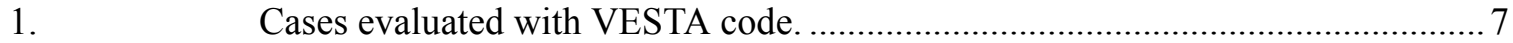

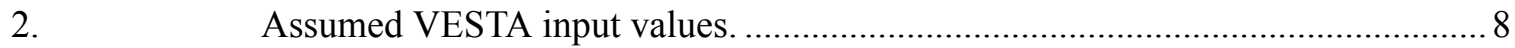

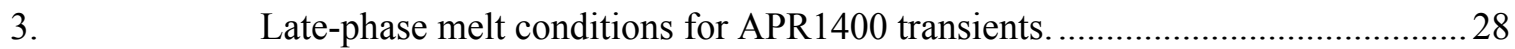

4. Relocation history for the APR1400 LOCA-1 transient. ...................................28 


\section{Introduction}

If cooling is inadequate during a reactor accident, a significant amount of core material could become molten and relocate to the lower head of the reactor vessel, as happened in the Three Mile Island Unit 2 (TMI-2) accident. If it is possible to ensure that the lower head remains intact so that relocated core materials are retained within the vessel, the enhanced safety associated with such plants can reduce concerns about containment failure and associated risk. For example, the enhanced safety of the Westinghouse Advanced $600 \mathrm{MWe}$ pressurized water reactor (PWR) (AP600), which relied upon external reactor vessel cooling (ERVC) for in-vessel retention (IVR), resulted in the U.S. Nuclear Regulatory Commission (US NRC) approving the design without requiring certain conventional features common to existing light water reactors (LWRs). IVR of core melt is therefore a key severe accident management strategy adopted by some operating nuclear power plants and proposed for some advanced LWRs. However, it is not clear that currently proposed ERVC without additional enhancements could provide sufficient heat removal for higher-power reactors (up to $1500 \mathrm{MWe}$ ). Hence, a three-year, United States (U.S.) - Korean International Nuclear Energy Research Initiative (INERI) project was initiated in which the Idaho National Engineering and Environmental Laboratory (INEEL), Seoul National University (SNU), Pennsylvania State University (PSU), and the Korean Atomic Energy Research Institute (KAERI) explored options, such as enhanced ERVC performance and an enhanced in-vessel core catcher (IVCC), that have the potential to ensure that IVR is feasible for higher power reactors.

\subsection{Program Objective and Tasks}

The ultimate objective of this INERI project is to develop specific recommendations to improve the safety margin for IVR in high-power reactors. The systematic approach applied to develop these recommendations combines state-of-the-art analytical tools and key U.S. and Korean experimental facilities. Recommendations focus on modifications to enhance ERVC (through vessel coatings to enhance heat removal and an improved vessel/insulation configuration to facilitate steam venting) and modifications to enhance in-vessel debris coolability (through improved IVCC configuration and materials). Collaborators use improved analytical tools and experimental data to evaluate options that could increase the margin associated with these modifications (see Figure 1). This increased margin has the potential to improve plant economics (owing to reduced regulatory requirements) and increase public acceptance (owing to reduced plant risk). This program is initially focusing on the Korean Advanced Power Reactor -1400 MWe (APR1400) design. However, margins offered by each modification will be evaluated such that results can easily be applied to a wide range of existing, advanced reactor designs, and next generation reactor (GEN IV) designs.

This three-year project includes four tasks (see Figure 2). In Task 1, which was completed during the first year of this research program, SCDAP/RELAP5-3D ${ }^{\complement}$ calculations were conducted to define representative bounding late phase melt conditions. Characteristic parameters from those bounding conditions (thermal loads, pressure, relocated mass, etc.) were used to design an optimized core catcher (in Task 2) and ERVC enhancements (in Task 3). Task 2 and 3 activities, which were initiated in the first year of this project, were completed during the third year of this project. In Task 4, which was completed during the third and final year of this project, collaborators assessed the improved margin obtained with Task 2 and 3 design modifications. Specifically, quantitative assessments were performed by re-evaluating the APR1400 scenarios analyzed in Task 1, considering Task 2 and 3 design recommendations. 

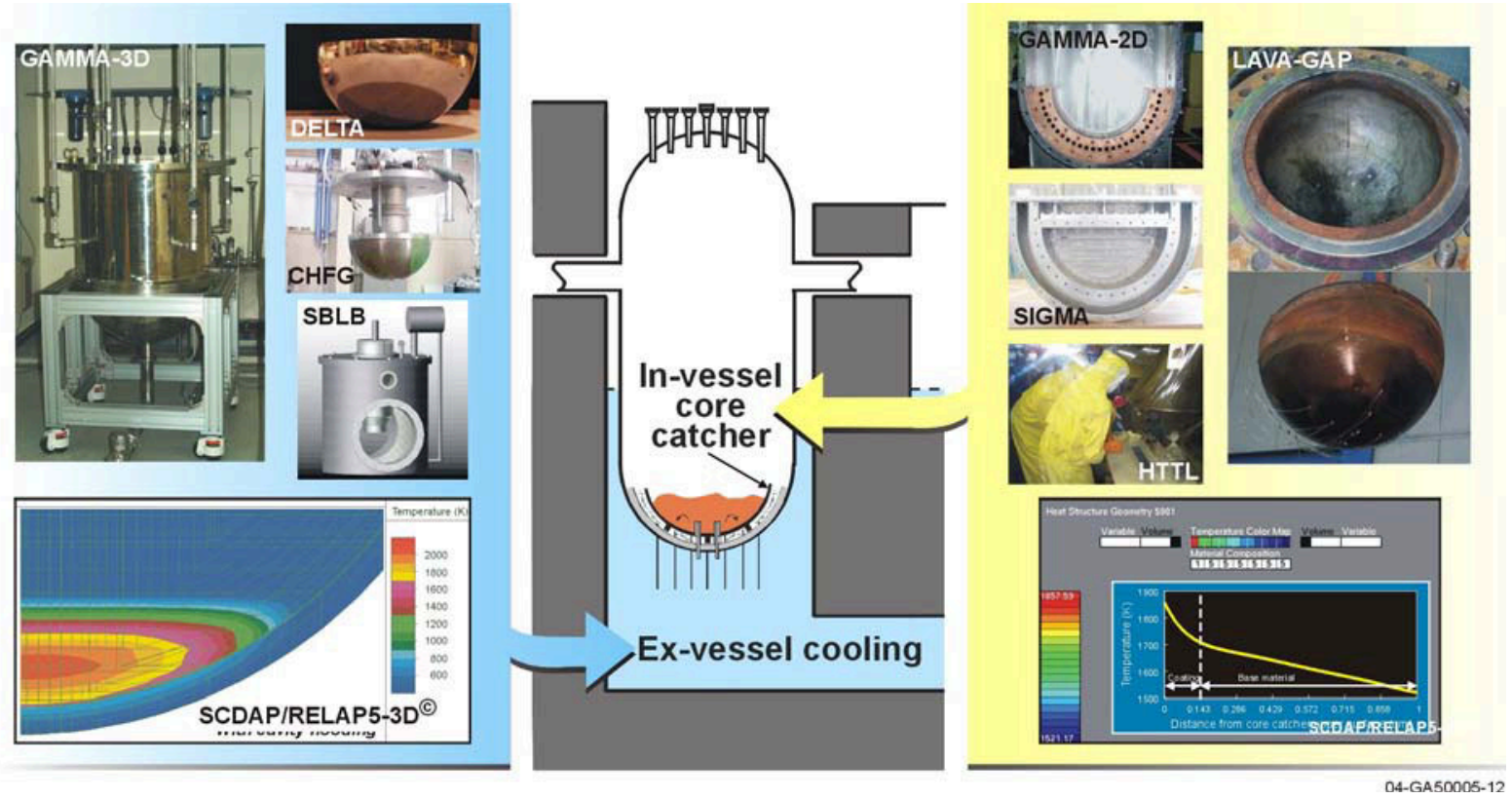

Figure 1. INERI applied key experimental facilities and state-of-the-art analytical tools.

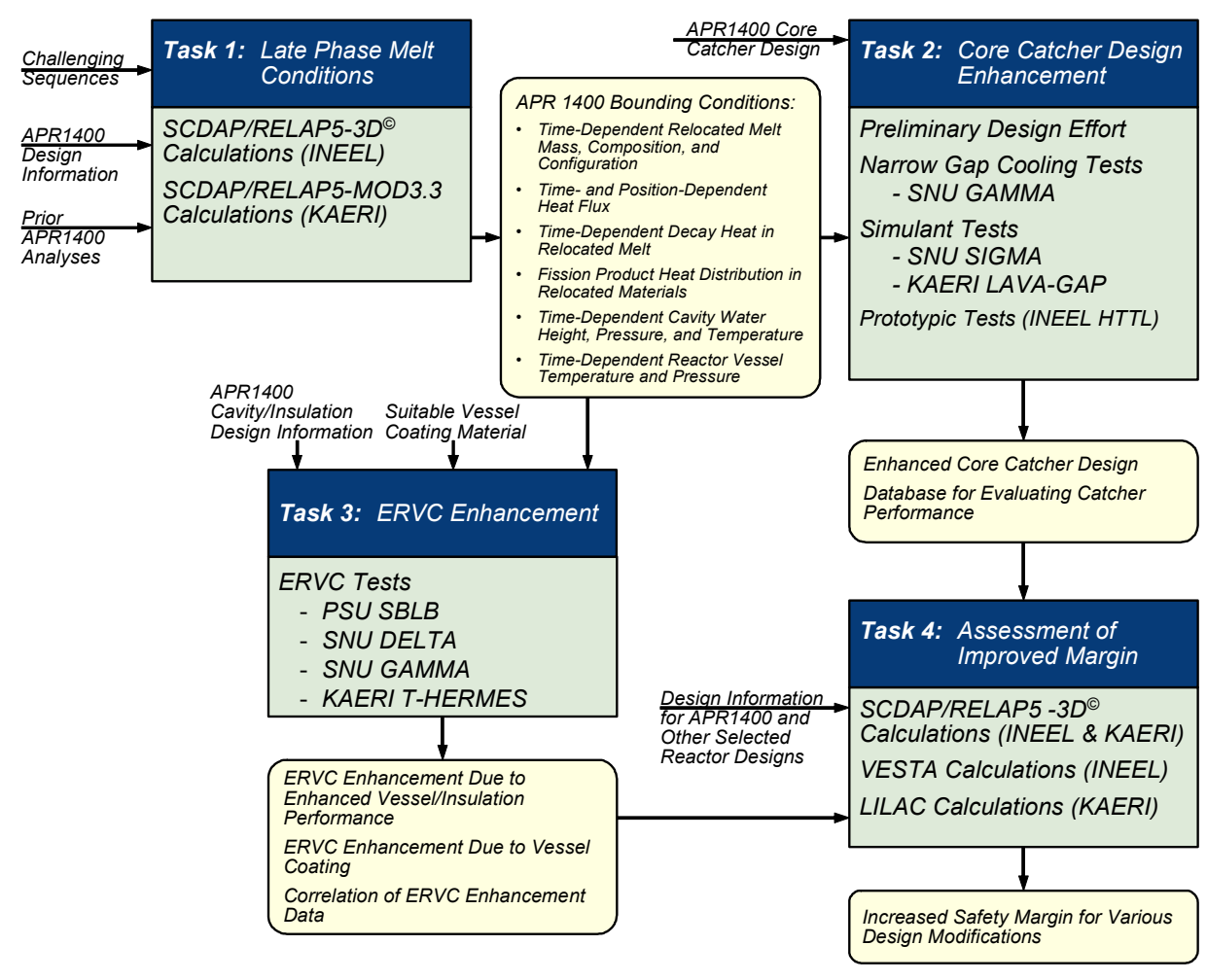

04-GA50005-13

Figure 2. Project organization illustrating key tasks and participating organizations. 
Results from Tasks 1, 2, and 3 can be found in several references (e.g., References 1 through 10). For the Task 4 evaluations, INEEL applied the SCDAP/RELAP5-3DC and VESTA codes to assess the improved margin associated with core catcher and ERVC design recommendations using results from Tasks 1,2 , and 3 . This report documents these INEEL calculations.

\subsection{Report Content}

This report presents results from INEEL VESTA and SCDAP/RELAP5-3D ${ }^{\odot}$ calculations to quantify increases in the margin for in-vessel retention that are associated with enhancements studied in this INERI project. VESTA calculations are described in Section 2, and SCDAP/RELAP5-3D ${ }^{\odot}$ calculations are described in Section 3. Section 4 contains insights and conclusions from these calculations. References cited in this report are listed in Section 5.

\section{VESTA Calculations}

The methodology, debris configurations, model description, input assumptions, and results associated with the VESTA calculations are outlined in the sections that follow.

\subsection{Methodology}

INEEL developed the VEssel Statistical Thermal Analysis (VESTA) code to independently verify University of California at Santa Barbara (UCSB) AP600 study results and assess the impact of additional uncertainties and other debris configurations. As documented in References 11 through 14, the VESTA model was developed with funding from the U.S. Nuclear Regulatory Commission (NRC) as a tool to assess the analyses presented for design certification of the Westinghouse AP600. ${ }^{15}$ As used here, this tool incorporated features that allowed evaluation of issues raised by peer-reviewers of the AP600 submittal.

\subsection{Debris Configurations}

A key assumption in assessing the heat load to the vessel from relocated debris is the configuration for the relocated material. At the time of the AP600 analyses, a FInal Bounding State (FIBS) was postulated that was supposed to "bound" thermal loads from any other configuration that can reasonably be expected. This FIBS assumed a molten ceramic pool lies beneath a metallic layer. The ceramic pool was assumed to contain all of the oxide core components (mainly $\mathrm{UO}_{2}$ and $\mathrm{ZrO}_{2}$ ). Heat transfer from such a pool is governed by turbulent natural convection associated with volumetric heat sources. The molten pool can experience sufficient cooling that it becomes surrounded by thin crusts that impose uniform temperature boundary conditions on the melt (i.e., its liquidus temperature). The metallic layer is also assumed to contain all unoxidized metallic components. It is heated from below and cooled from above and its sides. However, only the side boundary temperature is fixed -- at the metallic layer liquidus temperature as long as some portion of the vessel wall remains intact.

However, as noted in Reference 11, several of peer reviewers of the AP600 design certification submittal noted that the assumed FIBS may not bound even the most plausible configuration for the stratifica- 
tion of the relocated material. Figure 3 illustrates two possible alternate configurations that were proposed in Reference 11 as more challenging than the FIBS.

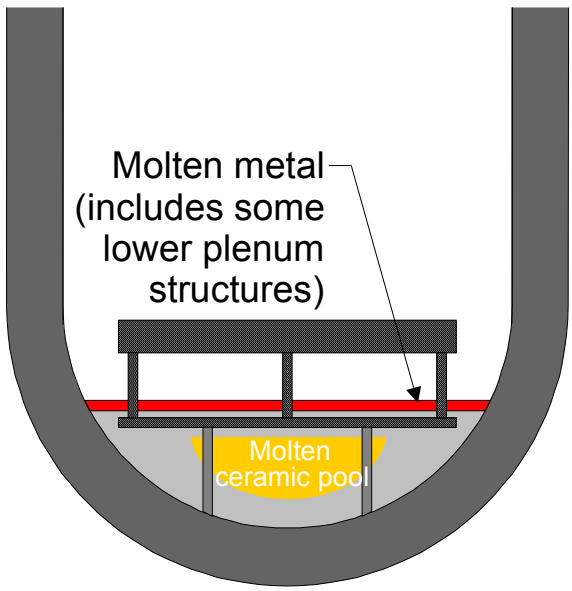

Configuration A

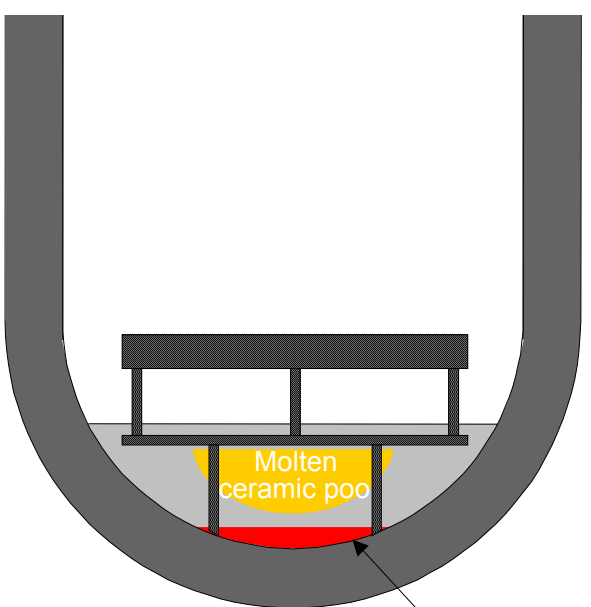

Molten metal (includes dissolved uranium in unoxidized zircaloy)

Configuration $\mathrm{C}$

Figure 3. Debris configurations evaluated for the APR1400.

Configuration A is similar to the FIBS, but it is evaluated at an earlier time period, before all of the metallic and ceramic material relocates to the lower head. In the AP600 analyses performed in the NRC review, melt relocation masses were based on SCDAP/RELAP5-3D ${ }^{\complement}$ calculation results. For the analyses conducted in this report, a similar reliance on SCDAP/RELAP5-3D ${ }^{\odot}$ results was employed. However, sensitivity calculations were also completed to assess the impact of the assumed metallic melt relocation mass.

Configuration $\mathrm{C}$ represents a case where sufficient uranium dissolves into unoxidized zirconium to form a heavier metallic layer. As noted in Reference 11, there were several source of data supporting U-Zr formation at the time that the AP600 underwent design certification. Since that time, data from the RASPLAV and MASCA tests also support the potential for such a configuration. ${ }^{16}$ As in the FIBS postulated for the AP600, this configuration assumes that more dense components have segregated from less dense components. However, the metallic layer sinks in this configuration. Heat loads are "focussed" on the bottom of the lower head where heat rejection is a minimum. ${ }^{5}$ VESTA calculations were then completed to assess the potential for IVR in this configuration.

\subsection{Code Description}

In VESTA, statistical distributions for the heat flux to the vessel wall from the molten pool are compared with statistical distributions calculated for the critical heat flux (CHF) from the submerged vessel surface. VESTA uncertainty distributions are Bayesian distributions, which are ultimately combined by a Monte Carlo sampling to yield a distribution on the probability of vessel heat fluxes exceeding the CHF (or if the vessel isn't submerged, the heat transfer rate from the vessel to the reactor cavity). VESTA can consider several types of debris configurations (stratified, uniform, etc.), decay heat power production associated with actinide and fission product heating, heat sources in the metallic material, and uncertainty 
distributions for a wide range of parameters. VESTA can be applied to obtain point estimates and probability density functions (pdfs).

The VESTA code considers the following heat transfer processes:

- steady-state turbulent natural convection within a volumetrically-heated (by decay heat), molten pool;

- convection within the metallic layer;

- conduction through the ceramic crust surrounding the molten pool and the vessel;

- radiation heat transfer from the upper surface of the top metallic layer to the inner surface of the upper plenum structures and the outer surface of the upper plenum structures to inner surface of the vessel;

- boiling heat transfer from the vessel outer surface (through an assumed outer surface temperature boundary condition).

As noted above, VESTA combines Bayesian uncertainty distributions by Monte Carlo sampling to yield a distribution on the probability of vessel wall heat fluxes exceeding the CHF. This is because it is presumed that vessel heat fluxes exceeding the CHF is a sufficient and necessary condition for vessel failure. Once the boiling crisis is reached, the bubble density becomes so large that bubbles coalesce, forming a vapor film that blankets the vessel surface. Although heat transfer may still occur via conduction and radiation across the vapor film, neither of these two processes is very effective. Hence, heat transfer coefficients would decrease significantly; and the temperature of the outer vessel wall would increase to the melt point or values where the steel essentially loses its strength and becomes susceptible to structural instability.

In addition, VESTA allows users to consider the following phenomena:

- several alternate debris configurations (see Section 2.2);

- decay heat power production associated with actinide and fission product heating;

- metallic layer heat sources based on actinide or fission product decay heat fractions and/or other heat sources;

- material property (density, specific heat, viscosity, thermal conductivity, volumetric coefficient of thermal expansion, eutectic temperatures) uncertainties and dependencies on temperature and/or composition;

- various decay heat loads uncertainty distributions (although VESTA includes a time-dependent decay power curve, the user may specific a decay power and its associated uncertainty distribution);

- various metallic layer heat transfer correlations and associated uncertainties (users may specific constants and associated uncertainty parameters for their desired correlations);

- various molten pool heat transfer correlations and associated uncertainties (users may specify constants and associated uncertainty parameters for their desired correlation for estimating average and angular dependent heat transfer coefficients);

- various CHF correlations and associated uncertainties (users may specify constants and associated uncertainty parameters for their desired CHF correlation); 
- various input parameter distributions (normal, log-normal, Student's t, uniform, user-specified, or point estimate) and characteristic uncertainty distribution parameters (median value, standard deviation, degrees of freedom, etc.).

VESTA modeling equations are listed in Appendix A of Reference 11. VESTA was verified for the FIBS and Configuration A endstates using information presented in the Reference 15 submittal by Westinghouse for the AP600 design certification.

Many of VESTA's capabilities were implemented so that this code could be used to address issues raised by peer reviewers of the AP600 design certification submittal. Hence, VESTA is an ideal tool for assessing the impact of various features explored in this INERI. Prior to applying VESTA for these calculations, several modifications were implemented into the Reference 11 code. First, VESTA was upgraded so that it could run under a Windows XP operating system. Second, an error in the Reference 11 version was corrected that affected Configuration $\mathrm{C}$ predictions. Results from the revised code for Configuration $\mathrm{C}$ were verified using hand calculations.

\subsection{Cases for Evaluation}

In this task, VESTA analyses were first completed for a "bounding" APR1400 base case for each configuration. As discussed in Section 1.1, SCDAP/RELAP5-3D ${ }^{\complement}$ calculations were completed in Task 1 of this project to identify an APR1400 "bounding case", considering a range of initiating events. To obtain "bounding" endstates, conservative assumptions were invoked related to plant response for the analysis of each event For example, one of the most conservative definitions was the assumed failure of all active safety injection systems. That assumption, along with others invoked in Task 1 analyses, yielded sequences that are expected to have very low probabilities for ever occurring. However, this conservative approach provided bounding late-phase melt conditions that could be used to quantify improvements in IVR margins that are achievable through the use of ERVC enhancements and the IVCC designed in this program.

Table 1 summarizes the cases evaluated with the VESTA code in this project. As discussed above, a key benefit of the VESTA code is that it allows users to consider the impact of relocated melt configuration. For this study, the two debris endstates shown in Figure 3 were evaluated. As shown in Table 1, several types of cases were evaluated for each debris endstate.

- A base case without an IVCC or enhanced ERVC.

- Bases case sensitivities to assumed steel relocation mass.

- Sensitivities that simulate the use of an IVCC (which was simulated by sensitivities to assumed decay heat).

- Sensitivities to various types of enhanced ERVC.

As noted above, the impact of an IVCC was simulated in these calculations by only considering the reductions in relocated material decay heat. Clearly, a detailed evaluation of the impact of this structure would require a thermal analysis that considered cooling of the relocated material contained within the IVCC (prior to any failure), the dilution in decay power density if the IVCC were to fail and become subsumed in the melt, and the decrease in heat flux to the vessel from relocated material due to delays associated with holdup in the IVCC. Because of project schedule constraints, it was only possible to consider decreases in decay heat associated with the increased time before relocating material comes in contact with the vessel. 
As discussed in Section 4, it is recommended that additional SCDAP/RELAP5-3D ${ }^{\complement}$ analyses be completed with newly obtained narrow gap cooling correlations to gain additional insights about the impact of the IVCC.

Table 1. Cases evaluated with VESTA code.

\begin{tabular}{|l|}
\hline \multicolumn{1}{|c|}{ Description of Cases } \\
\hline Configuration $\boldsymbol{A}$ - Stratified with Metallic Layer on Top \\
\hline Without IVCC or enhanced ERVC \\
Without IVCC or enhanced ERVC - sensitivities to assumed steel relocation mass \\
Enhancements that simulate the use of an IVCC (sensitivities to assumed decay heat) \\
Enhancements to ERVC (micro-porous coating, enhanced insulation, combined) \\
\hline Configuration $\boldsymbol{C}$ - Stratified with Ceramic Layer on Top \\
\hline $\begin{array}{l}\text { Without IVCC or enhanced ERVC } \\
\text { Without IVCC or enhanced ERVC - sensitivities to assumed steel relocation mass } \\
\text { Enhancements that simulate the use of an IVCC (sensitivities to assumed decay heat) }\end{array}$ \\
\hline
\end{tabular}

\subsection{Input Assumptions}

Table 2 lists VESTA input assumptions for these calculations. For many parameters, input was specified that was consistent with values assumed in the NRC review of the Westinghouse submittal. For example, coefficients required to simulate natural convection heat transfer from the molten pool were selected that were consistent with values obtained from tests conducted in support of the AP600 reactor evaluation.

Because of project schedule constraints, it was not possible to consider newly obtained heat transfer correlations to simulate natural convection heat transfer in a volumetrically heated pool. As discussed in Section 4 , it is recommended that additional SCDAP/RELAP5-3D ${ }^{\complement}$ analyses be completed with these newly obtained correlations (although initial evaluations suggest that these new correlations are similar to those used in the AP600 evaluations). 
Table 2. Assumed VESTA input values.

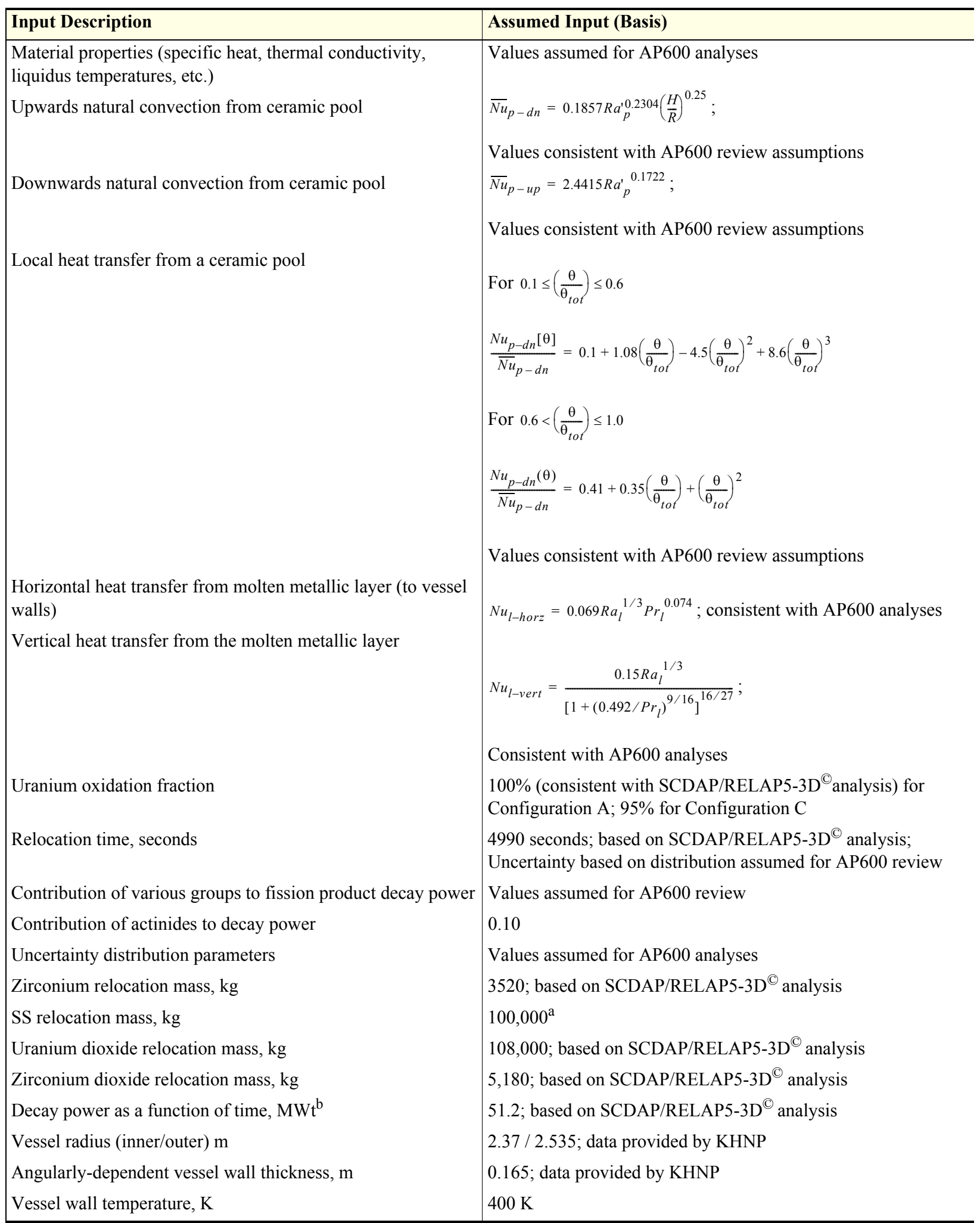


Table 2. Assumed VESTA input values.

\begin{tabular}{|c|c|c|c|c|c|}
\hline Input Description & \multicolumn{5}{|c|}{ Assumed Input (Basis) } \\
\hline Upper plenum structure surface area, $\mathrm{m}^{2}$ & \multicolumn{5}{|c|}{$\begin{array}{l}179.5 \mathrm{~m}^{2} \text {; base case values estimated by ratioing AP } 600 \text { masses } \\
\text { by power levels of the APR } 1400 \text { and the AP } 600\end{array}$} \\
\hline Maximum temperature of ceramic layer molten material, $\mathrm{K}$ & \multicolumn{5}{|l|}{2850} \\
\hline Cavity water temperature & \multicolumn{5}{|l|}{$400 \mathrm{~K}$} \\
\hline \multicolumn{6}{|l|}{ Vessel External Heat Transfer } \\
\hline $\mathrm{CHF}$ & \multicolumn{5}{|c|}{$\begin{array}{l}\text { Analyses conducted for uncoated, coated, insulated, and coated } \\
\text { with insulated conditions. For each case, a correlation was } \\
\text { implemented of the form: } \\
q_{C H F}^{\prime \prime}(\theta)=C_{1}+C_{2} \theta+C_{3} \theta^{2}+C_{4} \theta^{3}+C_{5} \theta^{4}, \mathrm{~W} / \mathrm{m}^{2} \\
\text { Correction uncertainties based on uncertainties assumed for } \\
\text { AP600 evaluation. }\end{array}$} \\
\hline Coefficients & $\mathrm{C}_{1}$ & $\mathrm{C}_{2}$ & $\mathrm{C}_{3}$ & $\mathrm{C}_{4}$ & $\mathrm{C}_{5}$ \\
\hline Plain vessel & $7.161 \mathrm{e}+05$ & $9.952 \mathrm{e}+03$ & $1.120 \mathrm{e}+01$ & $-1.416 \mathrm{e}-01$ & $0.000 \mathrm{e}+00$ \\
\hline Vessel with microporous coating & $1.412 \mathrm{e}+06$ & $-3.266 \mathrm{e}+04$ & $1.398 \mathrm{e}+03$ & $-1.072 \mathrm{e}+01$ & $1.297 \mathrm{e}-02$ \\
\hline Vessel with enhanced insulation & $1.277 \mathrm{e}+06$ & $3.218 \mathrm{e}+04$ & $1.095 \mathrm{e}+03$ & $-2.933 e+01$ & $1.837 \mathrm{e}-01$ \\
\hline Vessel with microporous coatings and enhanced insulation & $1.394 \mathrm{e}+06$ & $2.733 \mathrm{e}+04$ & $1.734 \mathrm{e}+03$ & $-4.252 \mathrm{e}+01$ & $2.592 \mathrm{e}-01$ \\
\hline
\end{tabular}

a. Base case values correspond to approximately $60 \%$ of masses that would be obtained by ratioing AP600 masses by power levels of the APR1400 to the AP600. Sensitivity studies were completed to evaluate the effects of this input value. b. Consistent with 1979 ANS 5.1 Standard and AP-600 analyses. Note that value was decreased in cases to evaluate the effects of an in-vessel core catcher.

As noted in Section 2.3, a key benefit of the VESTA code is that it allows users to consider the impact of input parameter uncertainties. Hence, the user must specify values to characterize median values and uncertainty parameters for calculation inputs. Table 2 summarizes the basis for the uncertainties assumed in VESTA calculations.

The use of microporous coatings and an enhanced vessel/insulation configuration were both investigated as methods to enhance ERVC in this INERI. As discussed in Reference 10, correlations were developed to predict heat transfer from the vessel for various heat transfer conditions. For the APR1400 bounding case heat transfer conditions and vessel geometry, the Reference 10 correlations were applied to obtain fifth order polynomial equations for estimating CHF correlations that could be implemented into VESTA. Table 2 lists the coefficients for the CHF correlations used in these VESTA calculations. Figure 4 compares the CHF correlations obtained for various ERVC conditions. As shown in this figure, the CHF may be increased by factors of 2 to 4 with proposed ERVC enhancements.

\subsection{Results}

As noted in Section 2.4, two debris endstate configurations were considered in these calculations. Results for Configuration A, a stratified configuration with an upper metallic layer, are discussed in Section 2.6.1; and results for Configuration $\mathrm{C}$, a stratified configuration with a lower metallic layer, are discussed in Section 2.6.2. Section 2.7 summarizes insights from these calculations.

VESTA output includes a wide range of variables, such as angularly-dependent crust thickness, angularly-dependent vessel thickness, peak, bulk, and average temperatures in the ceramic and metallic pools, 


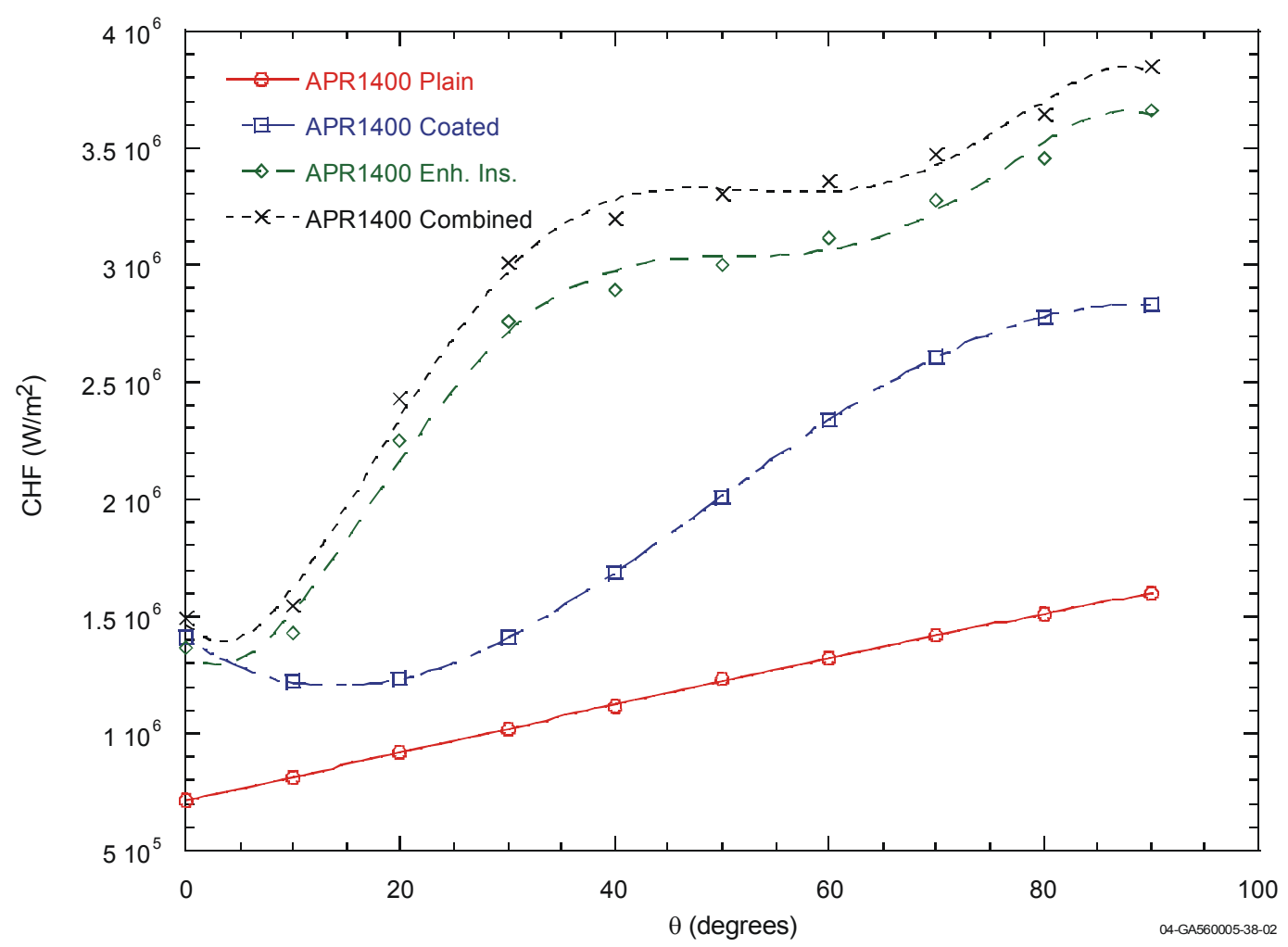

Figure 4. CHF associated with various ERVC conditions.

crust temperature, and core barrel temperature. However, results here focus on VESTA predictions for heat flux and ratios of this vessel heat flux to the CHF because these variables best reflect IVR margins.

\subsubsection{Configuration A - Stratified with Upper Metallic Layer}

\subsubsection{Base Case - without in-vessel core catcher or enhanced ERVC}

For this configuration, VESTA calculations were first performed for a "base case" that corresponded to the relocation conditions predicted by Task 1 SCDAP/RELAP5-3D ${ }^{\complement}$ results. As discussed in Section 2.5 , input values characterizing material properties, molten pool heat transfer via convection, and metallic pool heat transfer via convection and radiation were primarily based on values assumed in the Reference 11 AP600 analysis.

Figures 5 through 7 contain selected VESTA results for this "base case". Figure 5 contains VESTA predictions for the heat flux to the vessel wall as a function of angle. As illustrated in this figure, heat fluxes to the vessel at locations adjacent to the ceramic pool (e.g., less than $70^{\circ}$ ) are significantly lower than heat fluxes to the vessel at locations adjacent to the metallic pool. This result is due to the presence of heat sources in the metallic pool in conjunction with the lack of an insulating ceramic crust that is present at locations near the ceramic pool.

Figure 6 shows the corresponding CHF ratios (e.g., the ratio of the heat flux to the vessel to the CHF) for this base case. For point estimates, base case values are calculated in VESTA by dividing the angularly 


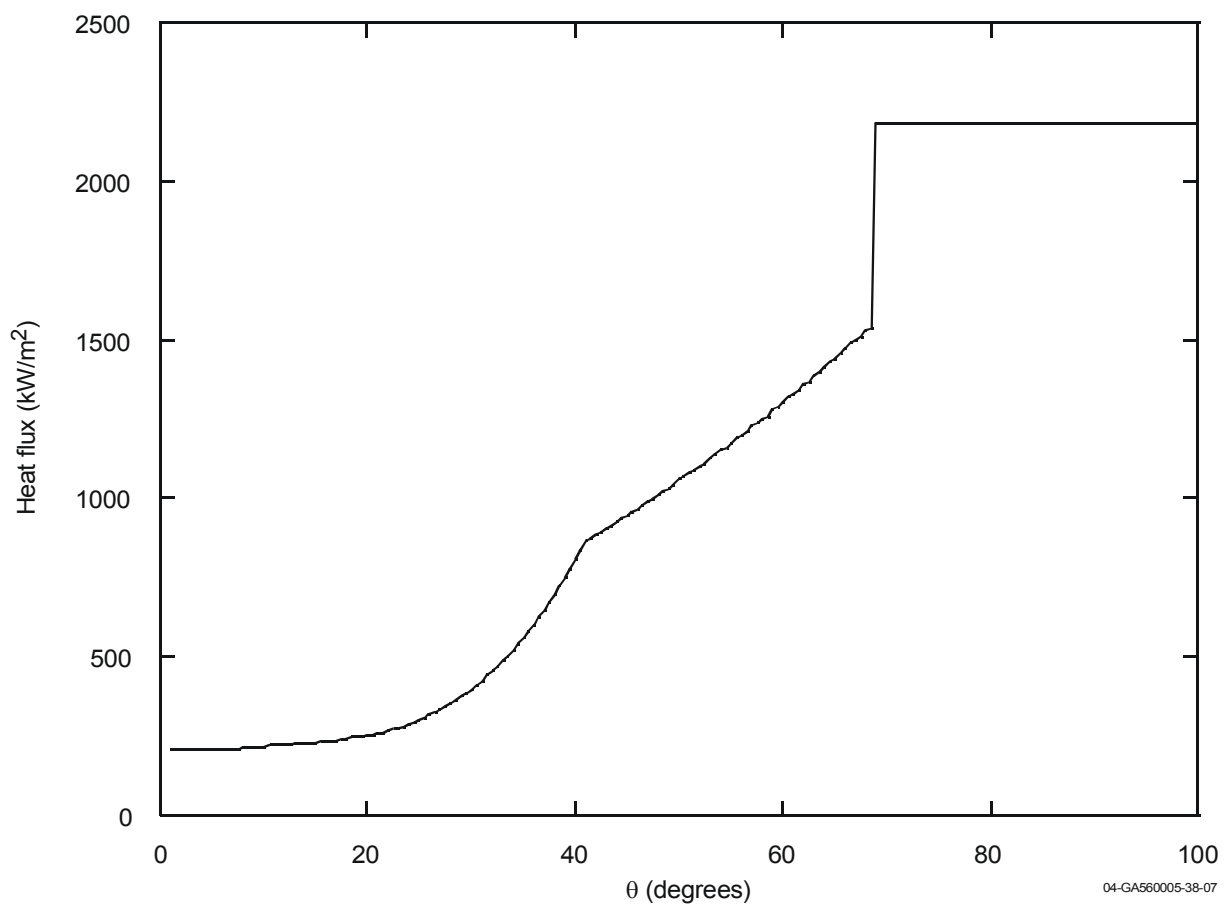

Figure 5. Heat fluxes to the vessel wall for Configuration A base case.

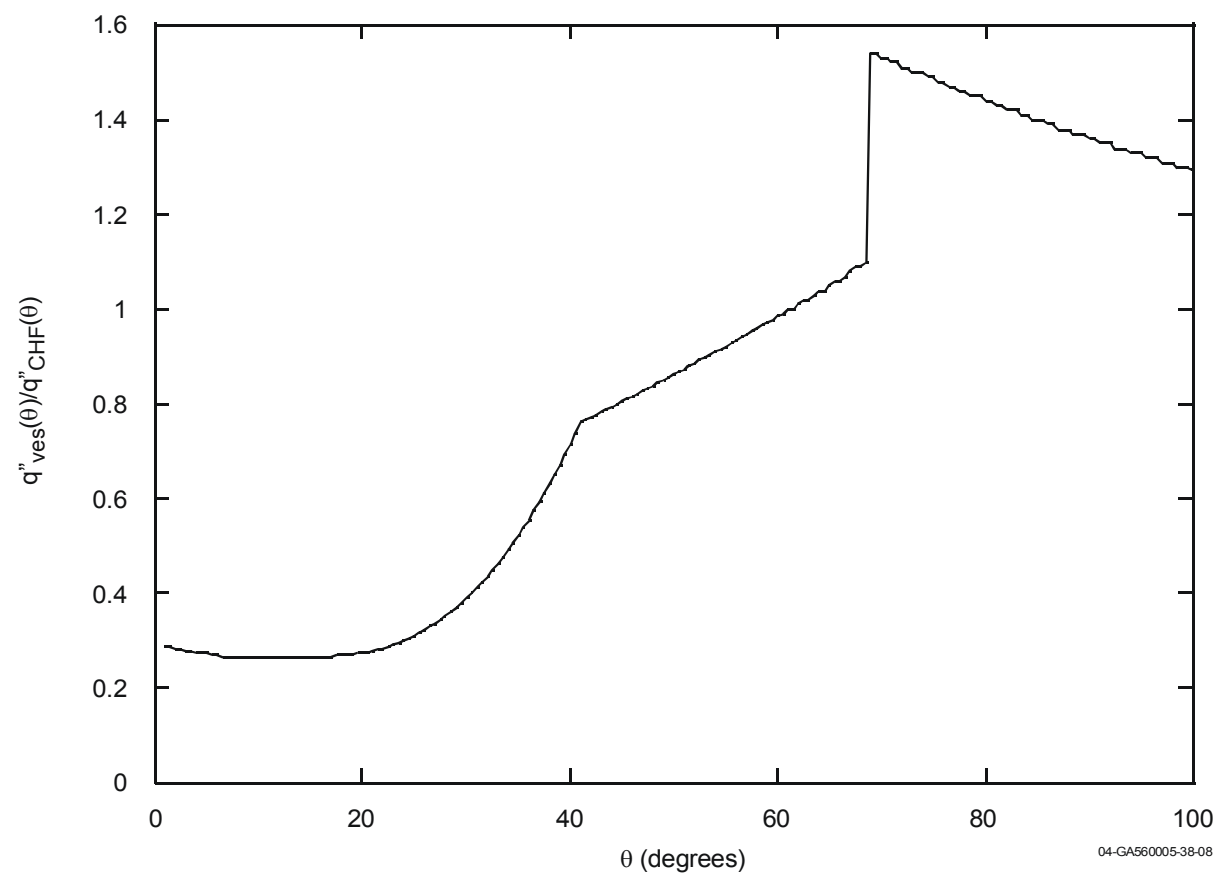

Figure 6. CHF ratios for Configuration A base case.

dependent heat fluxes in Figure 5 by CHF values for the plain vessel shown in Figure 4. As shown in Figure 5, vessel wall heat fluxes are predicted to exceed CHF values near the top of the ceramic pool (at an angle of approximately $70^{\circ}$ ) and at all locations adjacent to the metallic layer. 


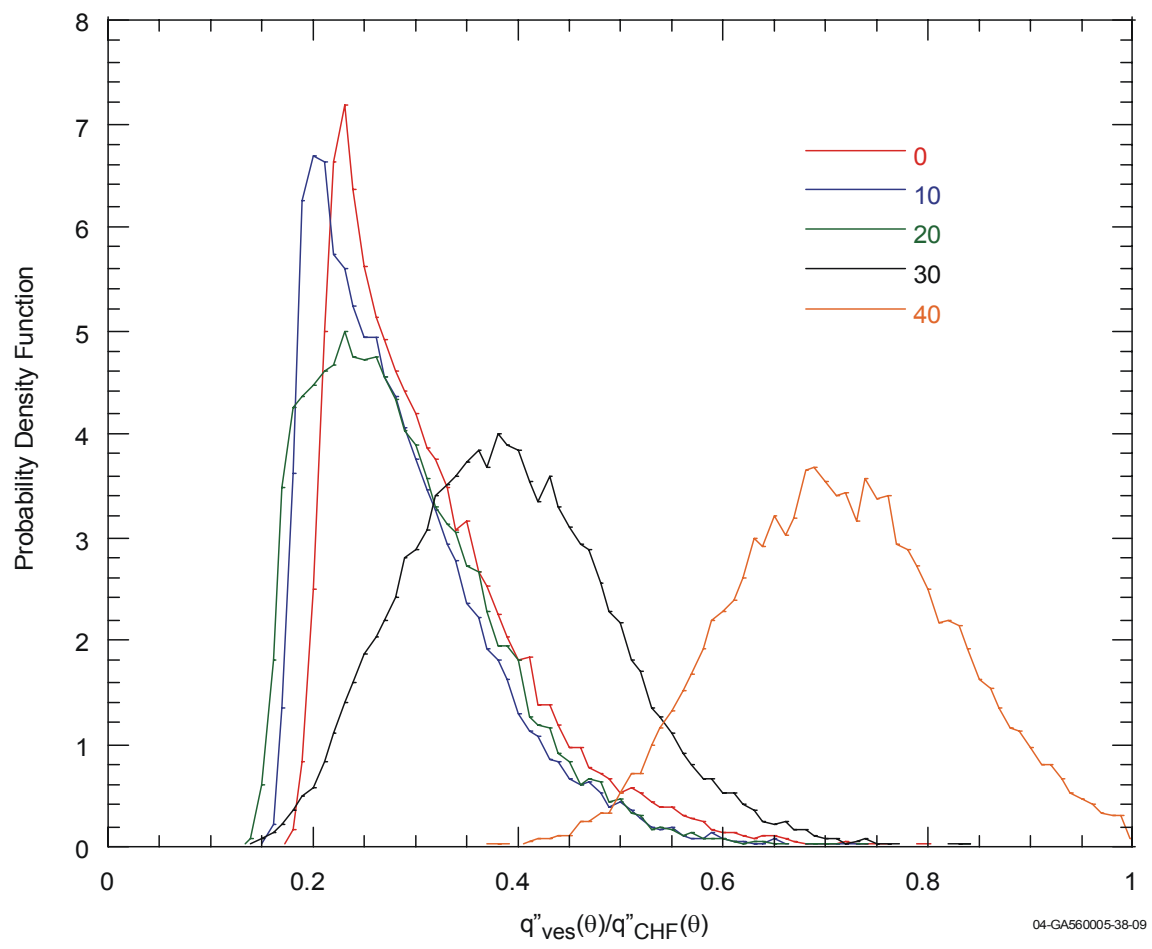

Figure 7. CHF ratio pdfs for Configuration A base case.

As noted above, the VESTA code allows users to assess the impact of uncertainties associated with various input values in its calculations. The code can present results in terms of cumulative distribution functions (cdfs) and probability distribution functions (pdfs). Figure 7 illustrates the pdfs for the ratios of vessel heat flux to the $\mathrm{CHF}$ at five locations that occur within the ceramic pool $\left(0,10,20,30\right.$, and $\left.40^{\circ}\right)$. As indicated in this figure, uncertainties in input values suggest that vessel wall heat fluxes may exceed unity at locations above $40^{\circ}$ (although point estimates suggest that median values remain below unity until a location of nearly $70^{\circ}$ ).

\subsubsection{Sensitivities to steel relocation mass}

As documented in Reference 11, one of the key assumptions in the Westinghouse submittal for the AP600 was the amount of steel mass assumed to relocate and form the metallic layer. Because steel melt mass assumptions significantly impact analysis results, a single pdf that encompasses the entire range of postulated melt masses possible at various times in the accident would not yield meaningful results. Hence, VESTA point estimate calculations were completed to assess the impact of the assumed steel relocation masses.

Results from these calculations are shown in Figures 8 and 9. As shown in Figure 8, variations in assumed steel relocation masses only affect metallic layer predictions because ceramic pool heat fluxes are independent of metallic layer mass assumptions. Although steel melt relocation mass assumptions affect metallic layer heat fluxes and associated CHF ratios, melt mass increases cannot reduce this ratio below 1.0 because it exceeds unity at locations adjacent to the ceramic pool (see Figure 9). 


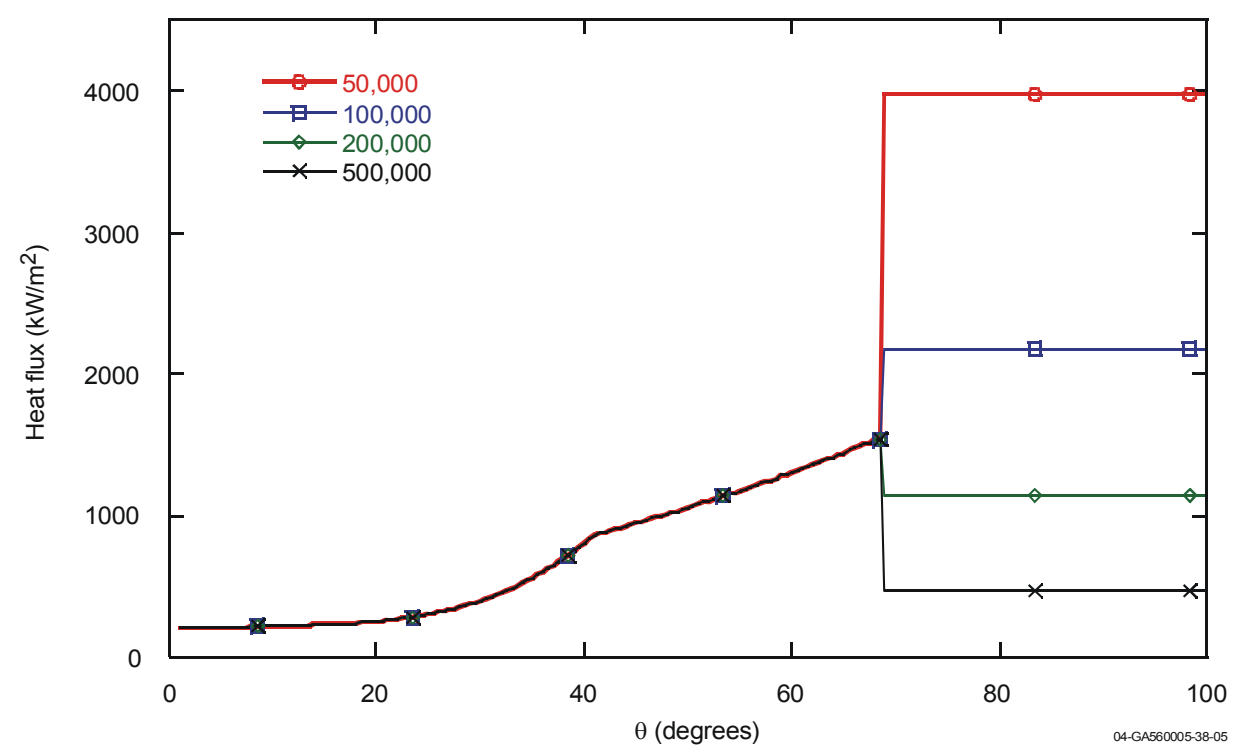

Figure 8. Heat fluxes assuming various metallic layer steel masses for Configuration A base case.

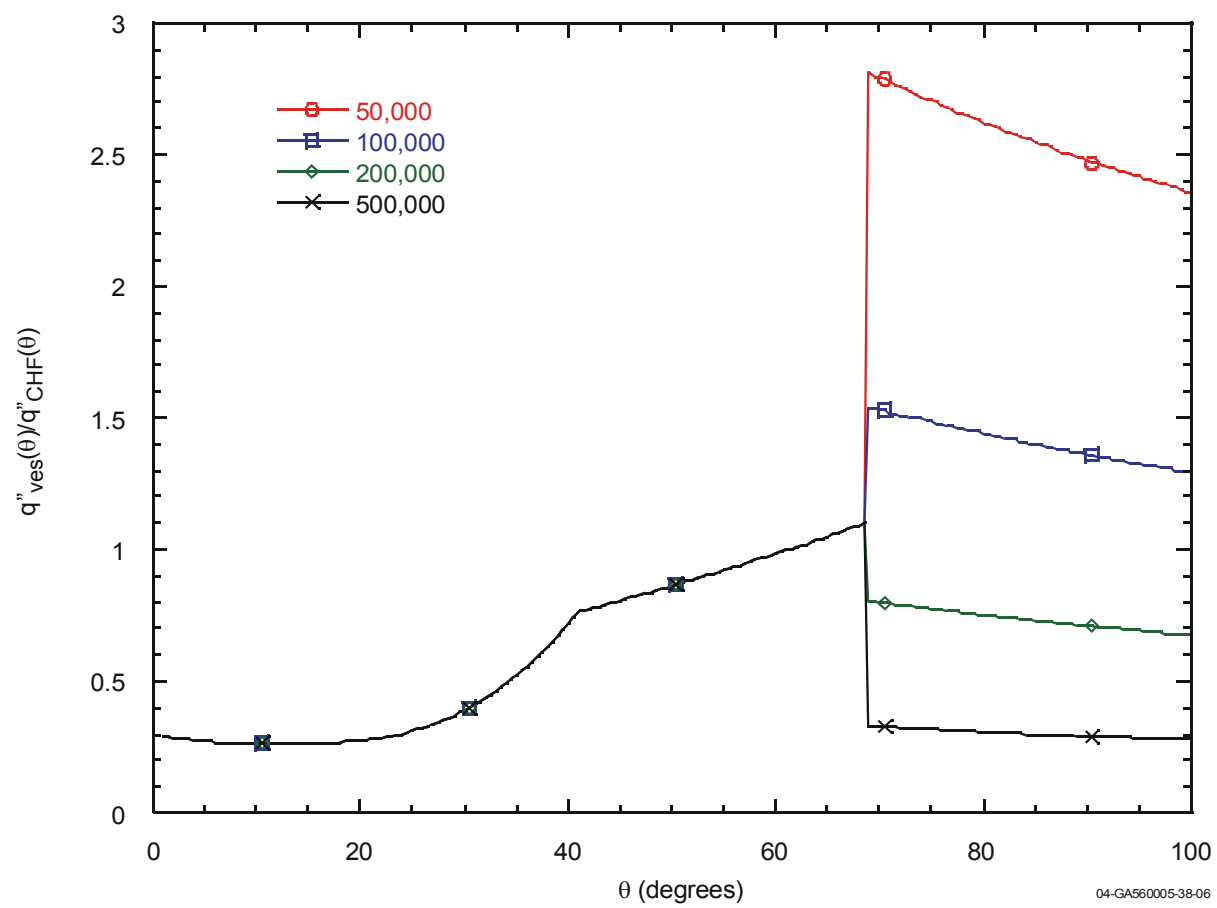

Figure 9. CHF ratios assuming various metallic layer steel masses for Configuration A base case.

\subsubsection{Enhancements to simulate an in-vessel core catcher}

As discussed in Reference 2, an IVCC may delay and/or prevent molten corium material from directly contacting the vessel wall due to the narrow gap cooling that occurs between the vessel and in-vessel core catcher, Furthermore, the decay heat in the relocated material is decreased, if the IVCC delays relocation of core materials to the lower head, or diluted, if the IVCC is subsumed into the relocated melt masses. 
Schedule constraints prevented detailed SCDAP/RELAP5-3D ${ }^{\complement}$ calculations from being conducted with the newly-obtained narrow gap cooling correlations to assess the impact of such a structure on the bounding case. However, VESTA sensitivity calculations were conducted to gain insights about the benefit associated with an in-vessel core catcher by considering the reduction in the magnitude of decay power if there is a delay in the time when the relocated material contacts the vessel wall. For these calculations, the decay power was reduced in increments consistent with $0.5 \mathrm{hr}, 1 \mathrm{hr}, 2 \mathrm{hr}$, and $4 \mathrm{hr}$ delays in melt relocation.

Figures 10 and 11 illustrate the reduction in heat flux and associated CHF ratios for each assumed relocation delay. As shown in Figure 11, a four hour delay in relocation is required to obtain point estimates for $\mathrm{CHF}$ ratios that approach unity. Although some additional reduction in decay heat may occur if the material associated with the IVCC is subsumed into the relocated corium, it is clear that the IVCC must survive for at least 4 hours in order to reduce vessel heat loads to values below the CHF for Configuration A debris conditions.

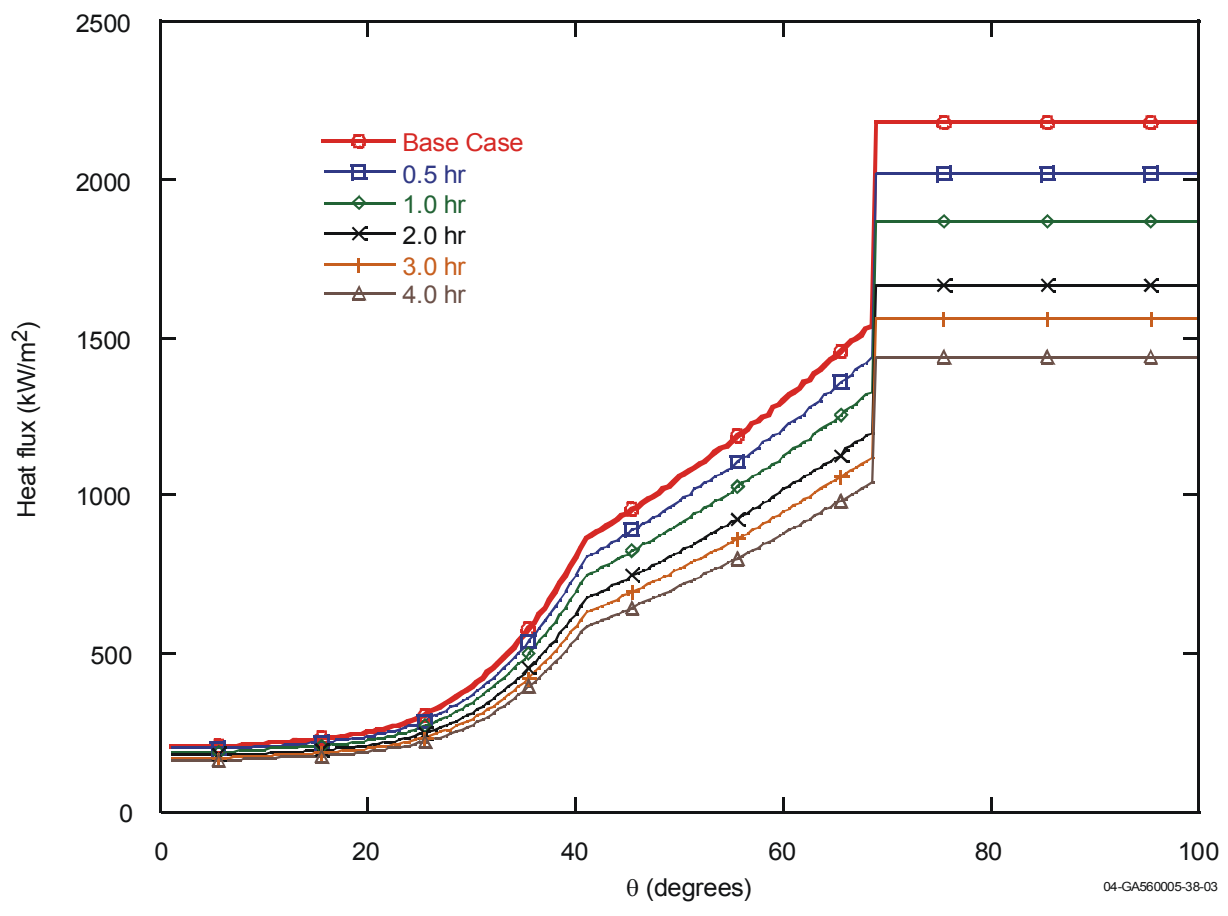

Figure 10. Heat fluxes assuming various relocation time delays for Configuration A base case.

\subsubsection{Enhancements to ERVC}

Figure 12 compares ratios of vessel heat flux to the CHF from point estimate calculations for proposed ERVC enhancements with values for a plain (uncoated and uninsulated) vessel. As shown in this figure, ratios are significantly decreased with proposed enhancements. Point estimate calculations suggest that vessel microporous coatings alone are sufficient to prevent vessel heat fluxes from exceeding the CHF. Even greater reductions in the CHF ratio are obtained with the enhanced insulation arrangement proposed in References 10. It is interesting to note that the proposed enhancements are not additive (e.g., the addi- 


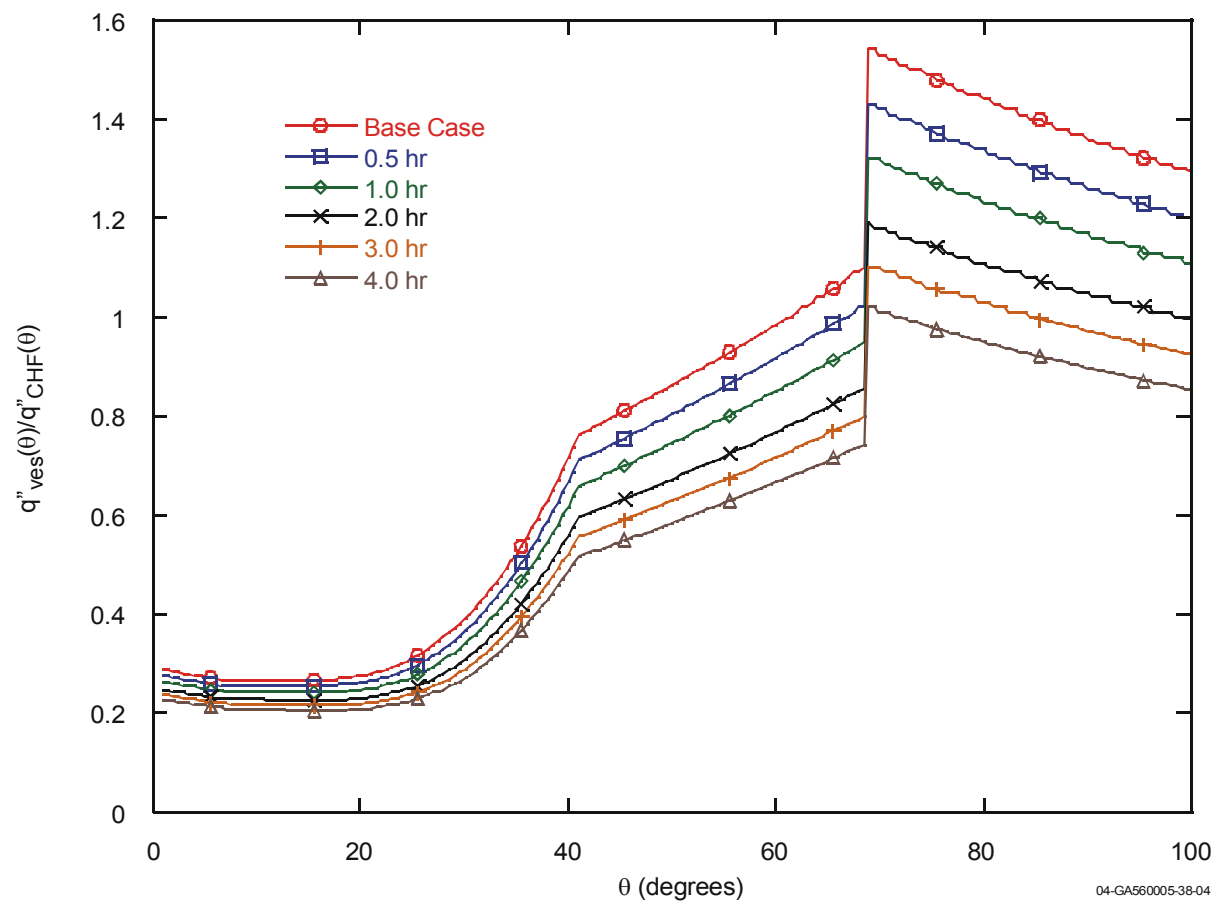

Figure 11. CHF ratios assuming various relocation times delay for Configuration A base case.

tional benefit of using microporous coatings on a vessel surrounded by the proposed enhanced insulation structure was smaller than the sum of the benefits associated with each option alone).

Results in Figures 13 through 15 suggest that the CHF ratios are below unity even when input uncertainties are considered. Note that in all three cases, uncertainty distributions are centered around the highest CHF ratios at locations near $70^{\circ}$. This result is consistent with results from the point estimate calculations presented in Figure 12.

\subsubsection{Configuration $\mathrm{C}$ - Stratified with Lower Metallic Layer}

\subsubsection{Base Case - without in-vessel core catcher or enhanced ERVC}

VESTA calculations were also performed for a Configuration C "base case" that corresponded to the relocation conditions predicted by Task 1 SCDAP/RELAP5-3D ${ }^{\complement}$ results. Figures 16 through 18 contain selected VESTA results for this "base case".

Figure 16 contains VESTA predictions for the heat flux to the vessel wall as a function of angle. As illustrated in this figure, heat fluxes to the vessel at locations adjacent to the ceramic pool (e.g., greater than $70^{\circ}$ ) are significantly higher than heat fluxes to the vessel at locations adjacent to the metallic pool.

As shown in Figure 17, point estimate calculations indicate that Configuration C CHF ratios remain below unity at all locations. However, the peak values for CHF ratios are predicted to occur at two loca- 


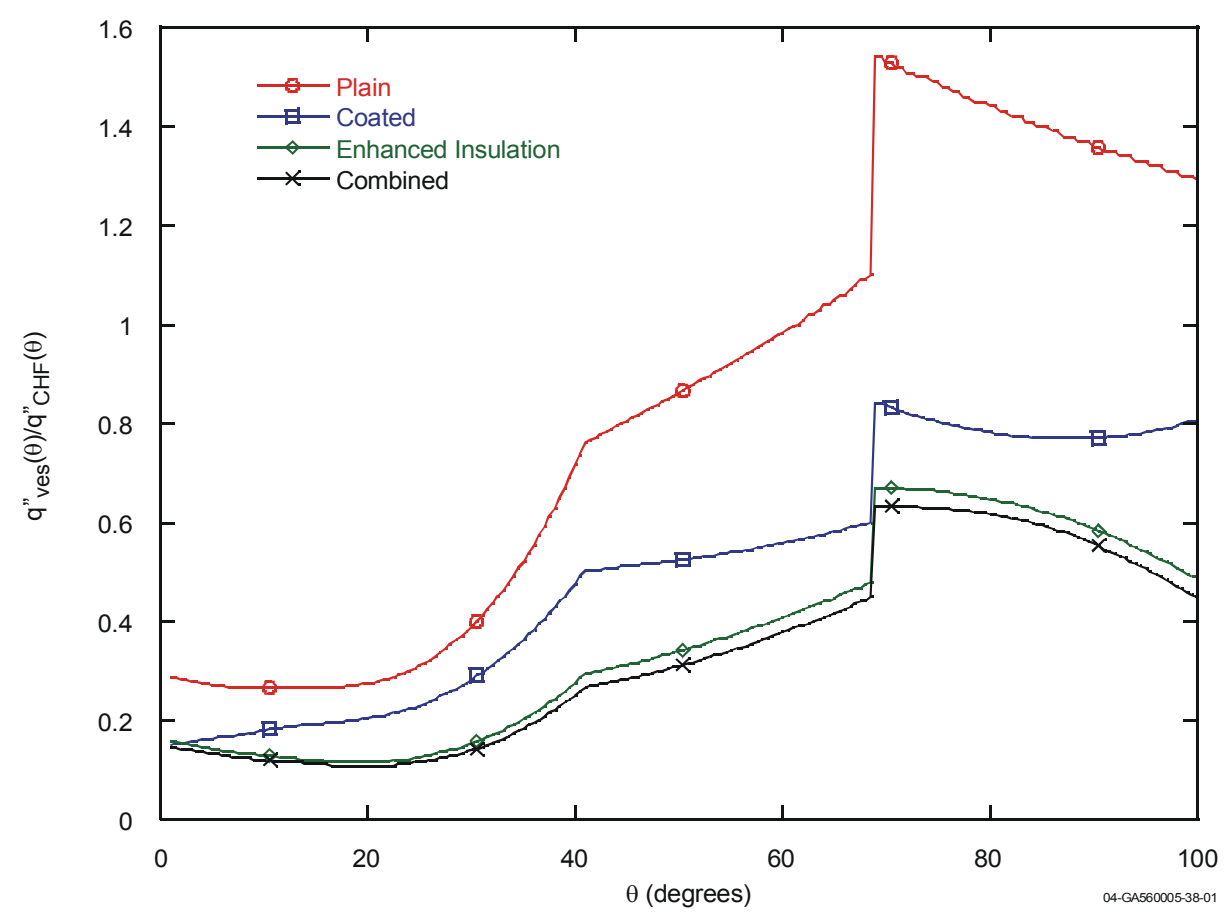

Figure 12. CHF ratios assuming various ERVC enhancements for Configuration A base case.

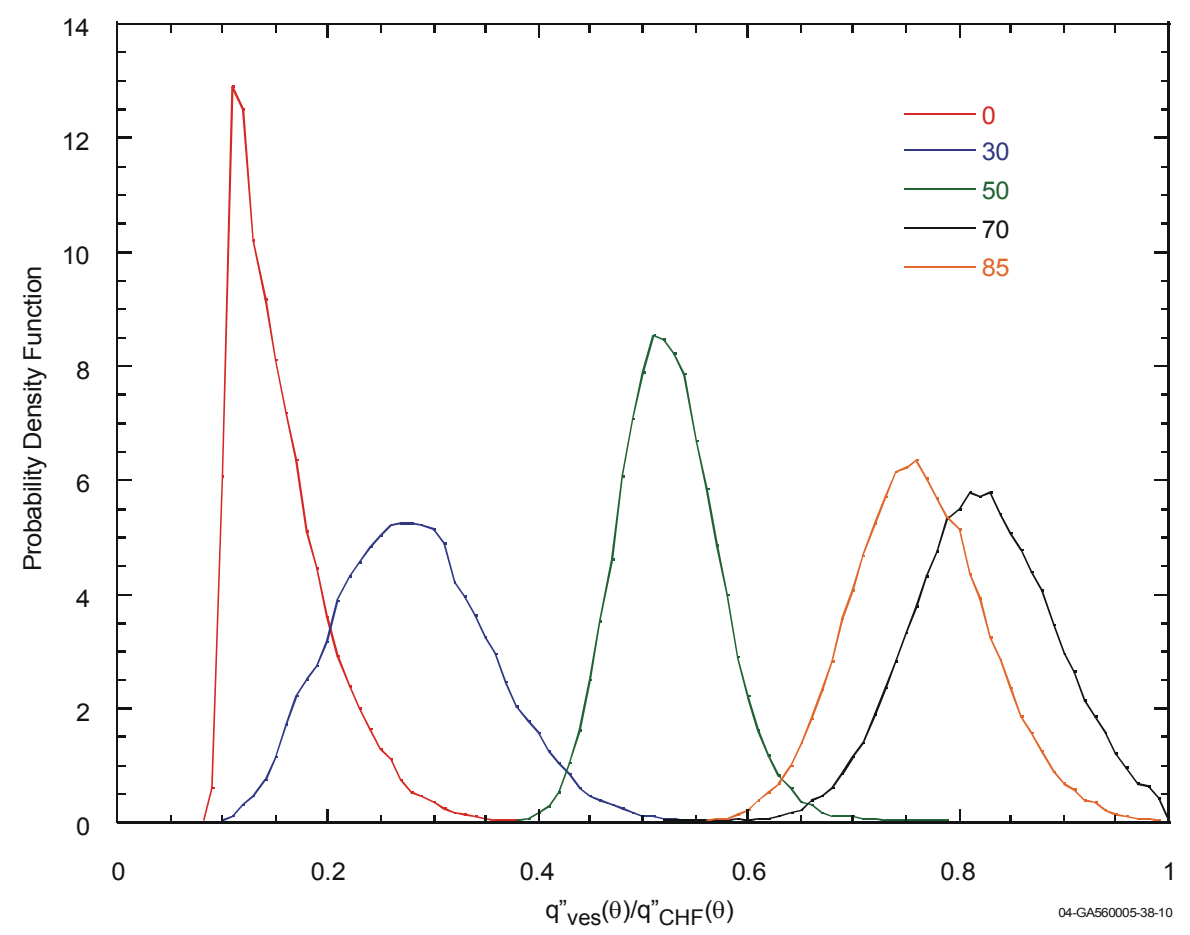

Figure 13. CHF ratio pdfs for Configuration A base case with vessel coating. 


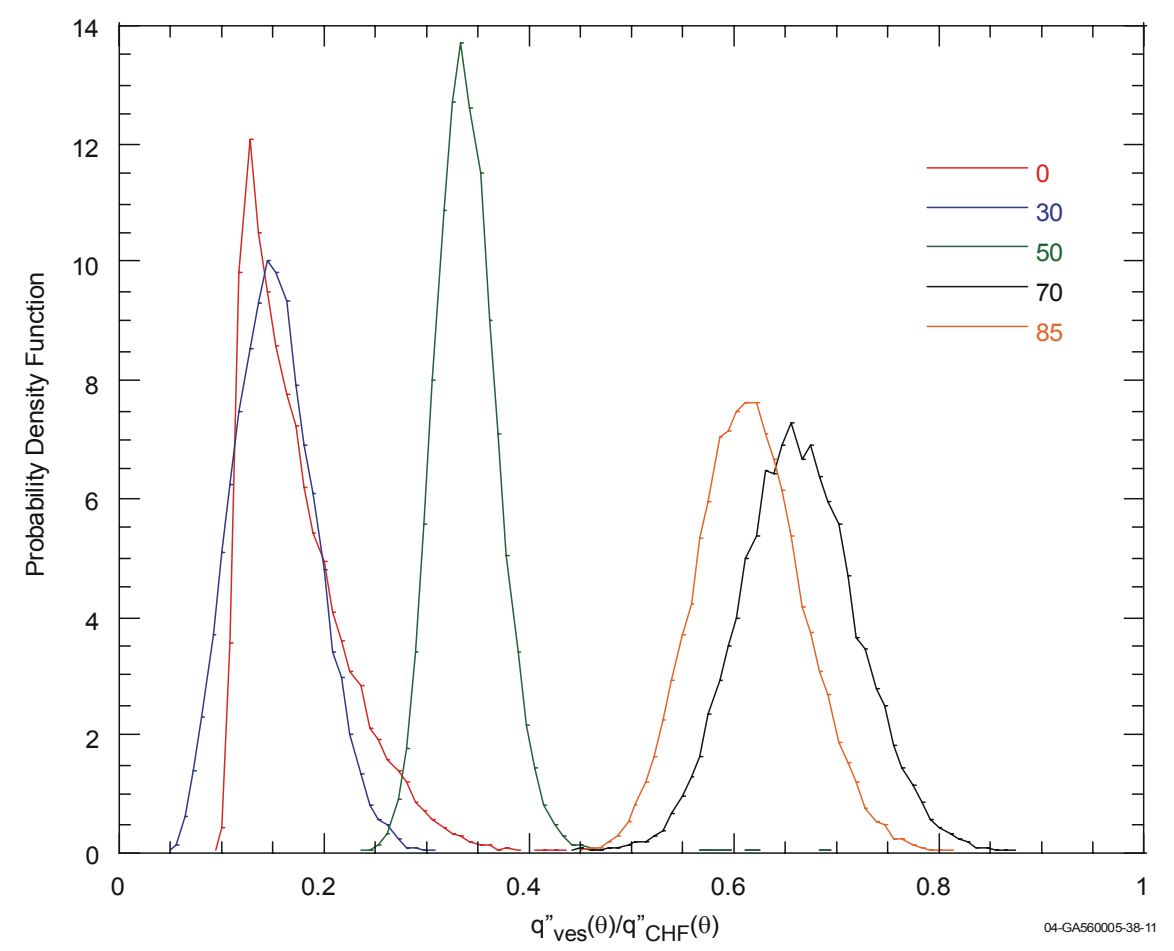

Figure 14. CHF ratio pdfs for Configuration A base case with enhanced vessel/insulation design.

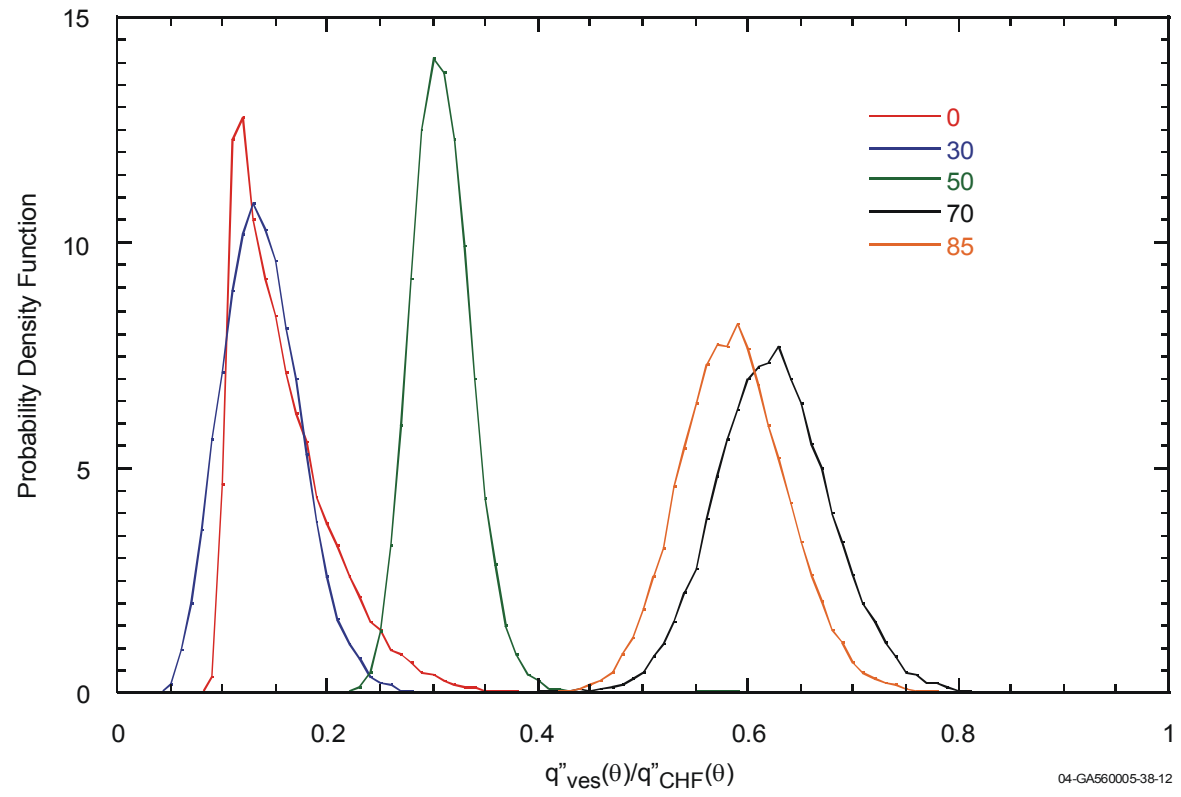

Figure 15. CHF ratio pdfs for Configuration A base case with "combined" ERVC enhancements. 


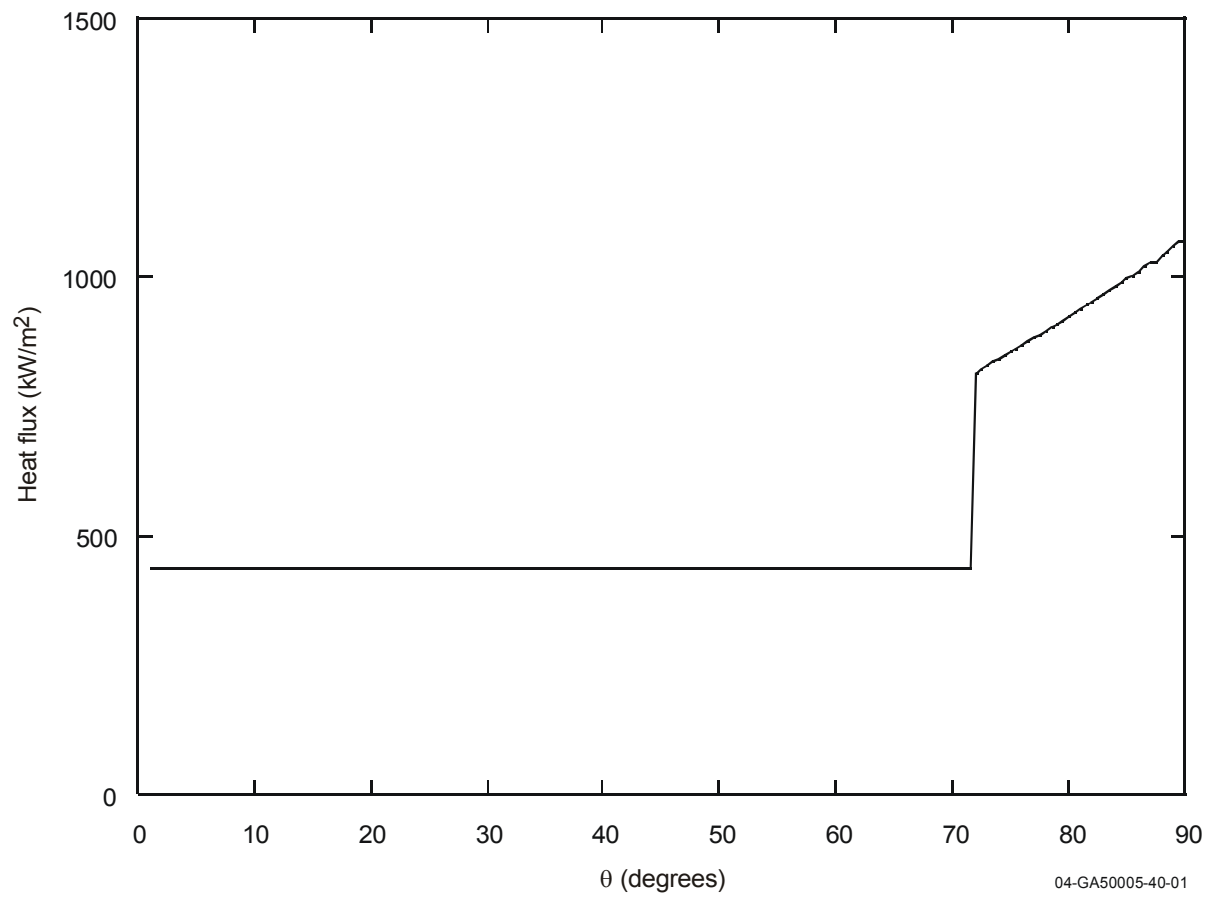

Figure 16. Heat fluxes to the vessel wall for Configuration $\mathrm{C}$ base case.

tions: near the bottom center of the vessel, where ratios are lower; and near the top of the ceramic pool, where heat fluxes to the vessel are higher.

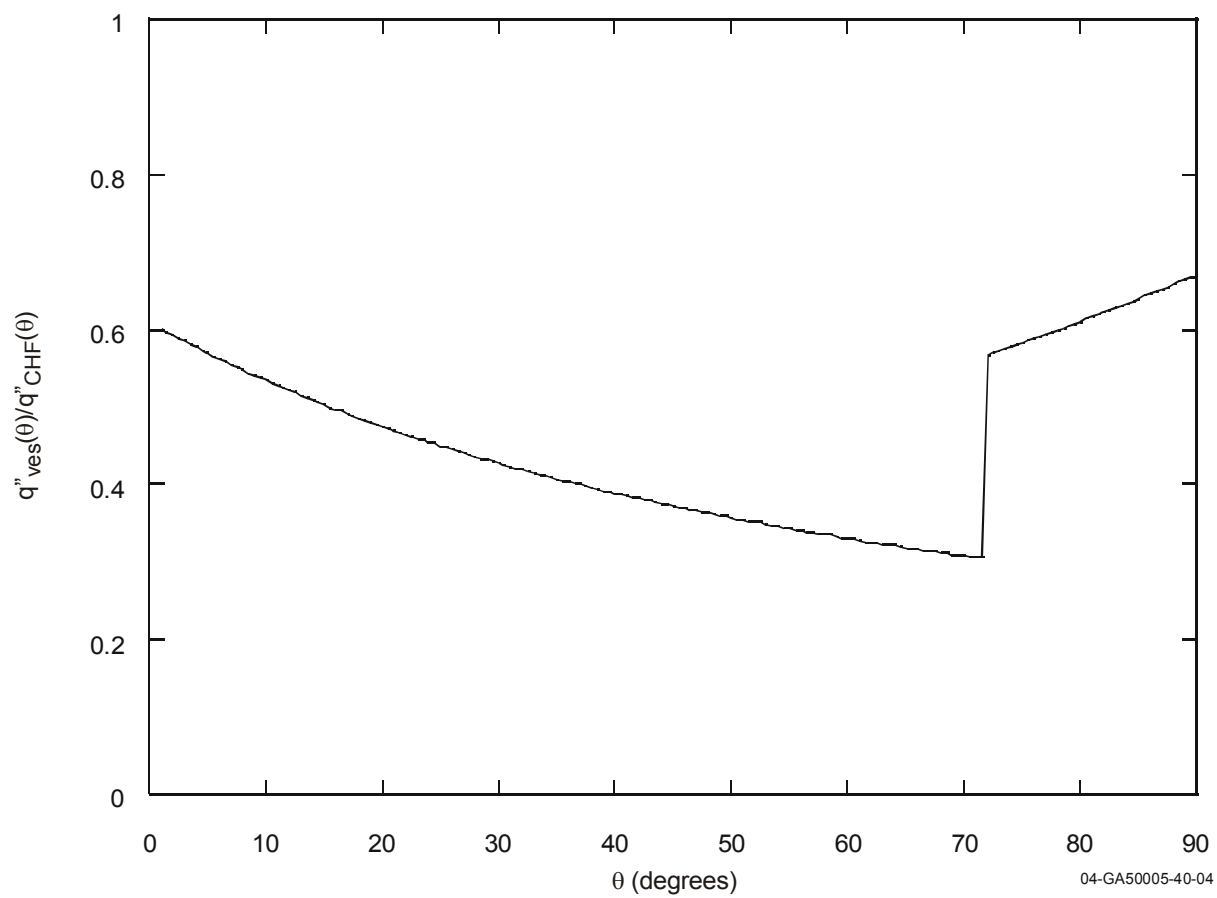

Figure 17. CHF ratios for Configuration $\mathrm{C}$ base case 


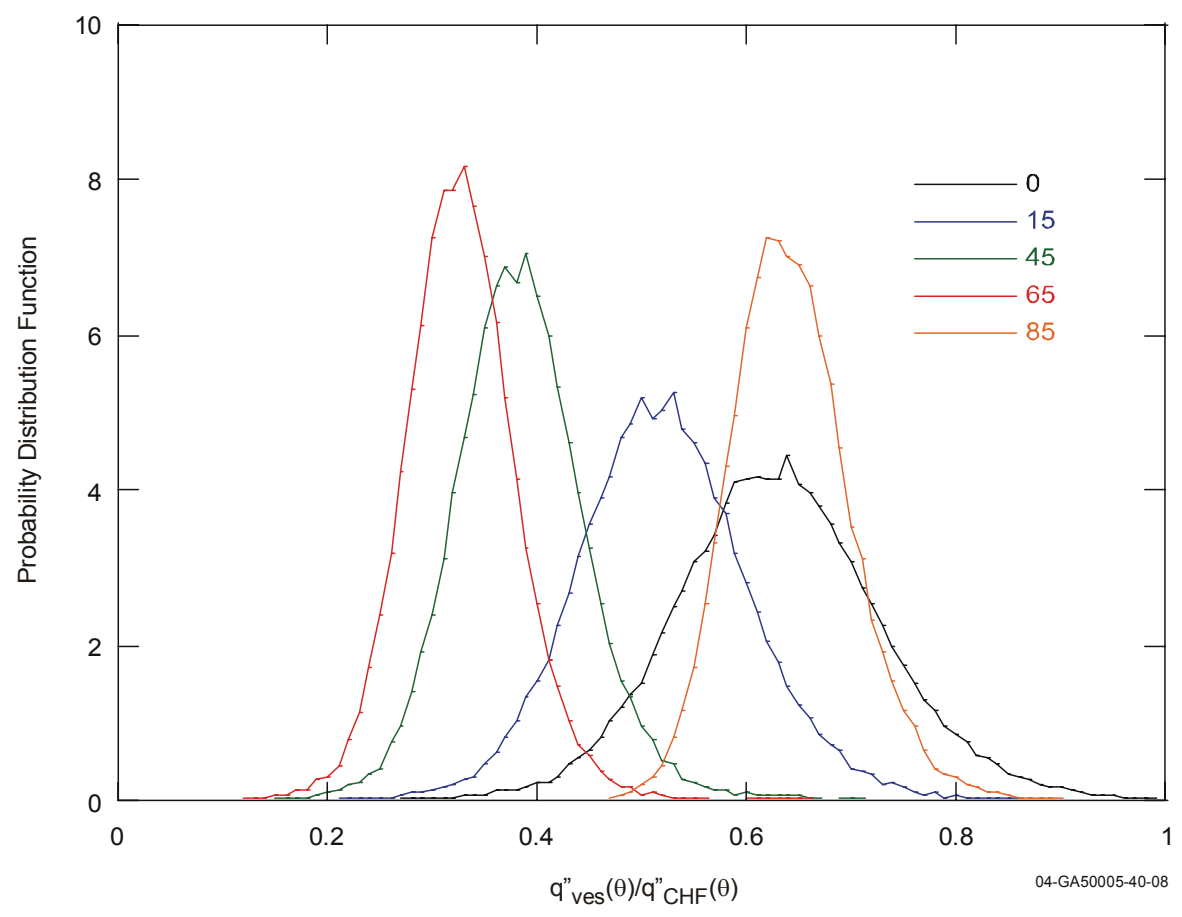

Figure 18. CHF ratio pdfs for Configuration $\mathrm{C}$ base case.

Figure 18 shows the pdfs for the CHF ratios at five locations that occur within the ceramic pool $(0,15$, 45,65 , and $85^{\circ}$ ). As indicated in this figure, the inclusion of uncertainties suggests that there is a very small, non-zero probability for Configuration $\mathrm{C}$ heat fluxes to exceed the $\mathrm{CHF}$ at two locations: at $0^{\circ}$ (near the bottom center of the vessel) and at $85^{\circ}$ (near the top of the ceramic layer).

\subsubsection{Sensitivities to steel relocation mass}

VESTA point estimate calculations were completed to assess the impact of the assumed steel relocation masses for Configuration C. Results from these calculations are summarized in Figures 19 and 20. As shown in Figure 19, a reduction in steel relocation mass for this configuration increases heat flux predictions at all angles. In the metallic layer, this is due to an increased power density when steel relocation mass is reduced. At locations above the metallic layer, the heat flux increases because these locations are now adjacent to the ceramic layer. As shown in Figure 20, the CHF ratios are also increased at all locations. Note that CHF ratios predicted with lower steel relocations masses yield peak CHF ratios near the bottom center of the vessel. Hence, a bottom center location of the vessel may be more susceptible to failure in such configurations.

\subsubsection{Enhancements to In-Vessel Retention}

VESTA sensitivity calculations were also conducted for Configuration $\mathrm{C}$ to gain insights about the benefits associated with IVCC and ERVC enhancements. For these calculations, the maximum benefits 


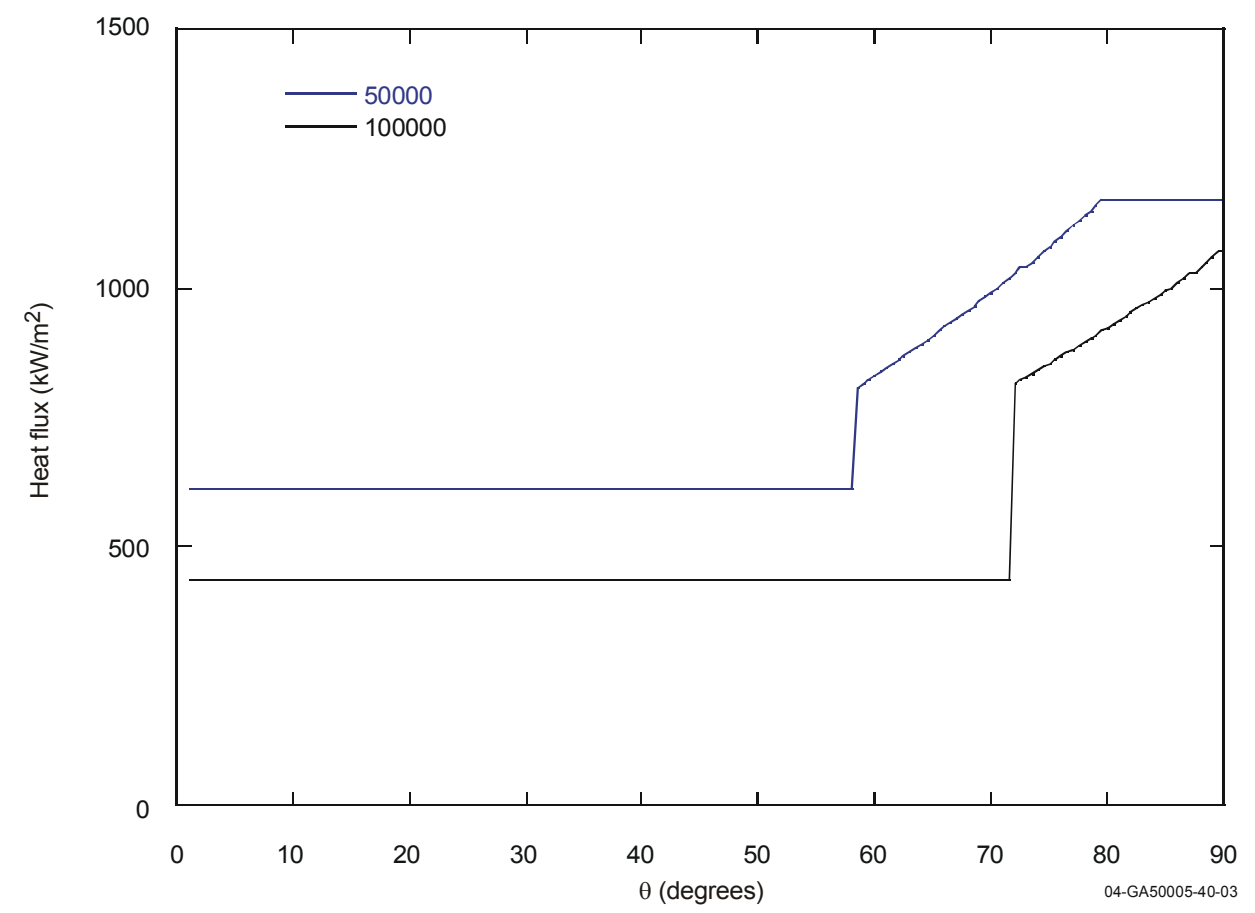

Figure 19. Heat fluxes for various metallic layer steel masses in Configuration $\mathrm{C}$ base case.

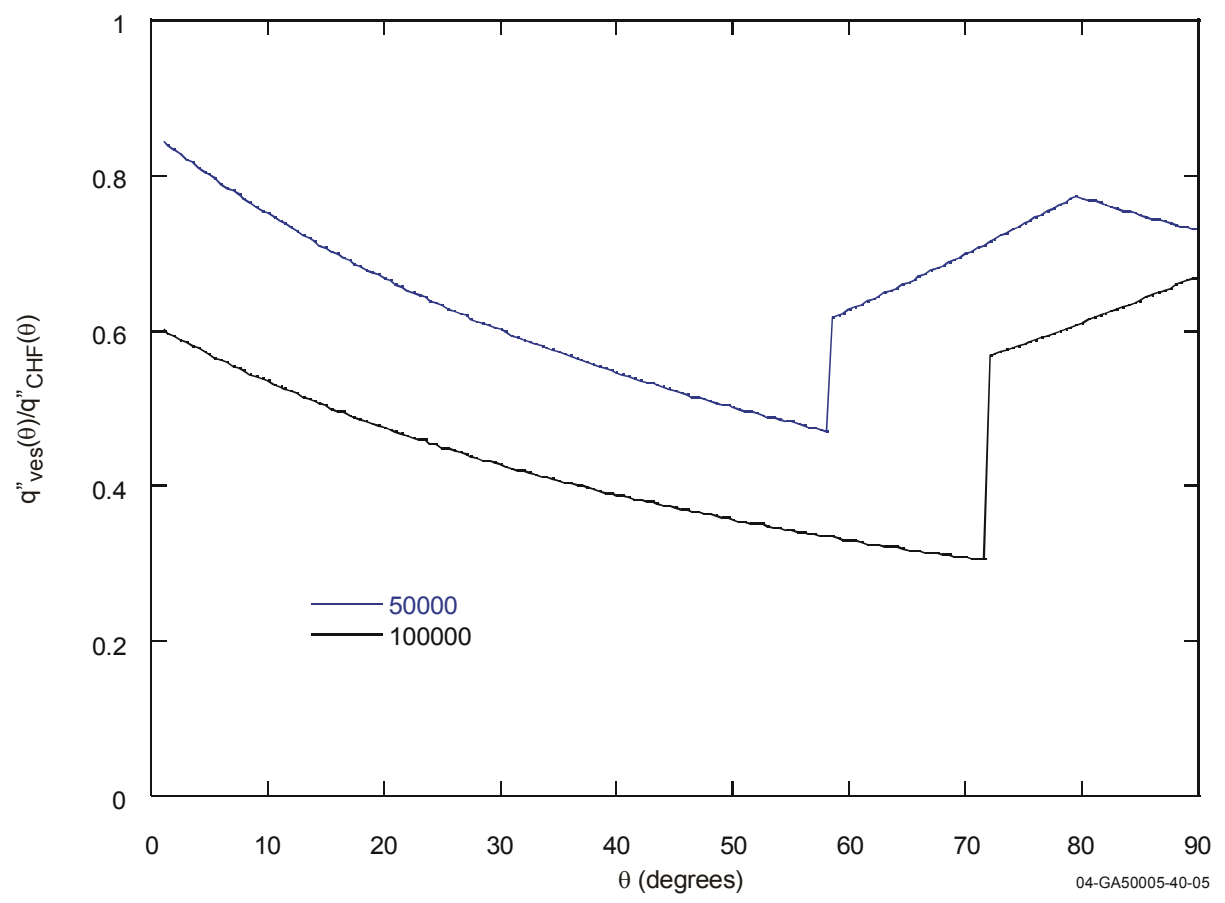

Figure 20. CHF ratios for various metallic layer steel masses in Configuration $\mathrm{C}$ base case.

explored for each IVR method was assumed. For example, an IVCC that delayed melt contact with the vessel for 4 hours and an "ERVC-Combined" case using a coated vessel with an enhanced insulation structure were assumed. As shown in Figure 21, both options significantly reduce CHF ratios. Uncertainty calcula- 
tion results suggest that CHF ratio pdfs remain below unity for both options. Although the reduction is generally greater for the "Combined" case with a coated vessel and an enhanced insulation structure, it is interesting to note that the IVCC offers the largest reduction at locations near the bottom center of the vessel, one of the locations of most concern in Configuration $\mathrm{C}$ endstates. Hence, the enhanced margin offered by various options is dependent upon the melt relocation configuration.

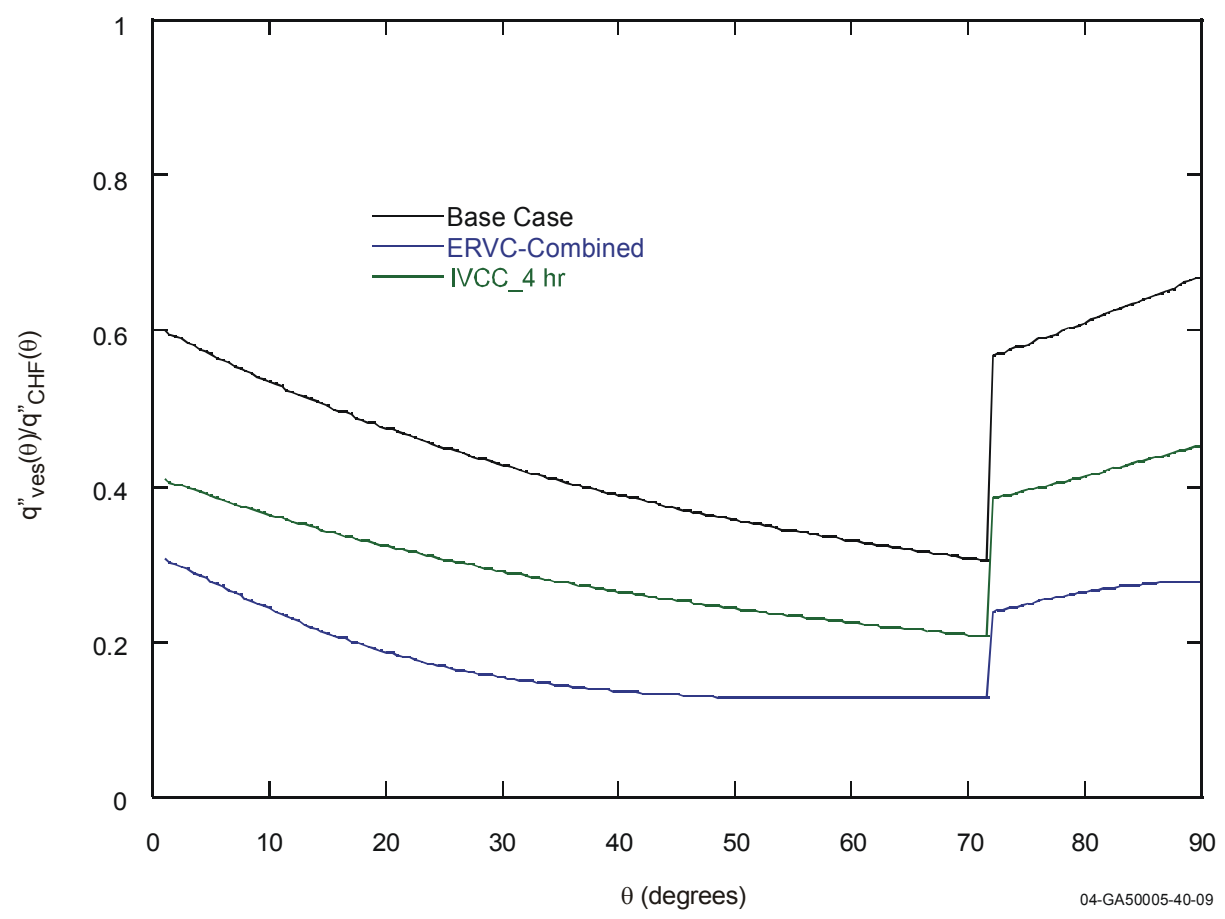

Figure 21. CHF ratios assuming various IVR enhancements for Configuration $\mathrm{C}$ base case.

\subsection{Summary}

VESTA calculations were completed for two debris endstate configurations to assess the impact of IVR enhancements considered in this INERI. Results for Configuration A, a stratified configuration with an upper metallic layer, and results for Configuration $\mathrm{C}$, a stratified configuration with a lower metallic layer, are discussed in Sections 2.6.1 and 2.6.2. This section summarizes insights from these calculations.

For base case conditions in a Configuration A endstate:

- Peak heat fluxes and associated CHF ratios are predicted to occur near the top of the ceramic pool beneath the metallic layer.

- If uncertainties in input values are considered, a small, but non-zero, probability exists for the CHF ratio to exceed 1.0.

- Point estimate calculations suggest that an IVCC must prevent melt relocation for at least 4 hours in order to maintain vessel heat fluxes below the CHF.

- Point estimate calculations suggest that either of the explored ERVC enhancements are sufficient to reduce vessel heat fluxes below the CHF. If one considers uncertainties, the additional reduction offered by an enhanced insulation design is required to maintain heat fluxes below the CHF. 
- An IVCC must decrease heat fluxes by at least a factor of 2 for this configuration.

- ERVC enhancements may decrease heat fluxes by factors of 2 to 5 .

For base case conditions in a Configuration $\mathrm{C}$ endstate:

- Higher heat fluxes are predicted to occur at vessel locations adjacent to the ceramic layer for this configuration. However, CHF ratios peak at two locations for this configuration: near the bottom center of the vessel and near the top of the ceramic pool.

- Point estimate and uncertainty calculations suggest that either an IVCC, which can prevent relocation onto the vessel for at least 4 hours) or a vessel with the combined ERVC enhancements considered in this study are sufficient to maintain vessel heat fluxes below the CHF.

In summary, either of the proposed IVR enhancements can preclude vessel heat fluxes from exceeding the CHF. Depending upon the enhancement selected and debris endstate configuration selected, IVR margins may increase by factors ranging from two to four. The benefit associated with each enhancement is dependent upon the debris endstate (a condition that is not possible to predict at this time).

\section{SCDAP/RELAP5-3D ${ }^{\odot}$ Calculations}

As previously noted, the objective of Task 4 in this INERI project is to use VESTA and SCDAP/RELAP5-3D ${ }^{\oplus 17}$ to evaluate potential improvements in the margins for successful IVR in high power reactors that may be achieved through use of an IVCC and ERVC enhancements (see Figure 2). For purposes of this project, APR1400 was used as a representative of high power reactors. Applications of VESTA to satisfy this objective were described in Section 2. This section contains a discussion of the corresponding SCDAP/RELAP5-3D ${ }^{\complement}$ efforts.

It is important to understand that SCDAP/RELAP5-3D ${ }^{\odot}$ provides a detailed analytical approach for predicting certain critical aspects of APR1400 behavior. While VESTA is well suited for completing a variety of sensitivity calculations, VESTA lacks the ability to simulate transient behavior and is limited by its treatment of boundary conditions. These kind of code-versus-code distinctions dictate the need for SCDAP/RELAP5-3D ${ }^{\odot}$. The possibility of APR1400 vessel wall thinning is a notable example. VESTA can be used to estimate a steady-state endpoint for wall thickness. However, SCDAP/RELAP5-3D ${ }^{\odot}$ provides the only means for simulating time-dependent vessel wall conditions. Depending on the balance between propagation of the thermal front from the molten corium and the rate of heat removal from the surface of the vessel, the actual wall thinning (which SCDAP/RELAP5-3D ${ }^{\complement}$ can approximate) may or may not correspond with the VESTA steady-state endpoint. For these, and other related reasons, SCDAP/RELAP5-3D ${ }^{\odot}$ calculations are needed for a complete IVR analysis.

\subsection{Methodology}

The approach used here essentially consists of making SCDAP/RELAP5-3D ${ }^{\odot}$ calculations of the APR1400 lower head thermal response following a 'limiting' molten corium relocation with and without an IVCC and ERVC enhancements. Comparing results from these calculations then allows determination of the potential IVR benefits that may be derived if some specific reactor design changes were actually implemented. 
Conditions defining the 'limiting' molten corium relocation were taken from SCDAP/RELAP5-3D ${ }^{\complement}$ results generated in Task 1 of this project. Calculations with and without an IVCC and ERVC enhancements were made possible by modifying SCDAP/RELAP5-3D ${ }^{\odot}$ to allow simulation of results from experiments conducted in Tasks 2 and 3. Those code modifications are described in some detail in Section 3.2, the associated modeling assumptions are outlined in Section 3.3, results are provided in Section 3.4, and a summary of the effort is given in Section 3.5.

Before proceeding, it should be noted that only lower head ERVC enhancement effects were considered relative to high power reactor IVR margins. IVCC was not addressed in any SCDAP/RELAP5-3D ${ }^{\complement}$ calculation due to schedule and budget constraints.

\subsection{Code Modifications}

ERVC enhancements that were considered included the effects of microporous coatings (to promote nucleation during the boiling process), the effects of an optimized vessel insulation configuration (to promote water downflow and steam venting), and the effects of microporous coatings combined with optimized vessel insulation. SCDAP/RELAP5-3D ${ }^{\complement}$ was modified to allow simulation of those (three) ERVC enhancement options based primarily on PSU experimental results collected under Task $3 .{ }^{10}$ In addition, PSU experimental results for a plain reactor vessel lower head (without coatings or insulation) ${ }^{10}$ were incorporated into the code to serve as a reference for gauging any potential IVR margin improvements. In all cases (for plain, coated, insulated, and coated/insulated vessels), the PSU experimental results consisted of correlations for the CHF (as a function of angular position and water subcooling) and correlations for nucleate boiling and transition boiling (as functions of angular position and the vessel wall superheat).

Correlations for the CHF were provided in the form

$q_{C H F}=f(\theta) f\left(\Delta T_{\text {sub }}\right)$

where

$q_{C H F}=$ the critical heat flux $\left(\mathrm{MW} / \mathrm{m}^{2}\right)$,

$\theta=$ the angle shown in Figure 22 (radians),

$\Delta T_{\text {sub }}=T_{\text {sat }}-T_{\text {water }}\left({ }^{\mathrm{o}} \mathrm{C}\right)$,

$T_{\text {sat }}=$ the water saturation temperature $(\mathrm{K})$, and

$T_{\text {water }}=$ the water temperature $(\mathrm{K})$.

Unlike the CHF, correlations for nucleate and transition boiling were provided only at discrete angular positions. However, at each discrete angular position studied, correlations were provided in the form

$q=a(\ln \Delta T)^{3}+b(\ln \Delta T)^{2}+c(\ln \Delta T)+d$

where

$q=$ the (nucleate or transition) boiling heat flux $\left(\mathrm{MW} / \mathrm{m}^{2}\right)$, 


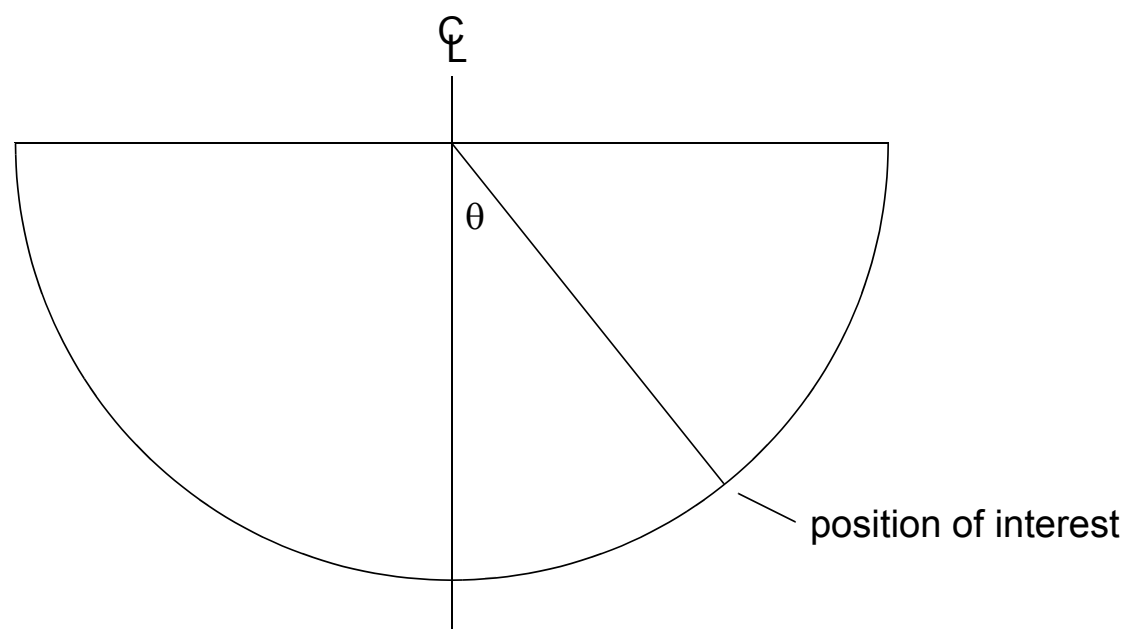

Figure 22. Illustration of an angular position relative to heat transfer from a hemispherical surface.

$\Delta T=T_{w}-T_{\text {sat }}\left({ }^{\circ} \mathrm{C}\right)$,

$T_{w} \quad=$ the vessel wall surface temperature $(\mathrm{K})$, and

$a, b, c$, and $d=$ correlation constants.

PSU results for a coated vessel at an angle of $0.98 \mathrm{rad}\left(56^{\circ}\right)$ is shown in Figure 23 as an example. In this figure, nucleate and transition curves are depicted for saturated boiling at atmospheric pressure. The CHF is also shown. SCDAP/RELAP5-3D ${ }^{\odot}$ was modified to allow the appropriate simulation of heat transfer corresponding with figure. Similarly, code modifications were also completed for all other angular positions studied in plain, coated, insulated, and coated/insulated configurations.

In all cases, the code modifications included transient treatment of subcooling effects. This was accomplished by calculating a saturation temperature at each time and angular position of interest consistent with the total pressure, which is the sum of the position-dependent hydrostatic head and the system pressure of the water surrounding the reactor vessel. The resulting time- and position-dependent saturation temperature allowed calculation of a corresponding time- and position-dependent CHF.

The time- and position-dependent CHF was then used to linearly scale nucleate and transition boiling curves in the modified code. This approach was used because the CHF was provided as functions of subcooling and position while nucleate and transition boiling curves were available only at a limited number of discrete positions under saturated conditions. Scaling was always applied to the nucleate and transition boiling curves from the nearest reported angular position (for the configuration being analyzed). This scaling approach retains the shape of the experimentally-determined boiling curves while ensuring consistency of the curves with the experimentally-determined CHF.

The code modifications also included heat transfer prior to the onset of nucleate boiling. This was accomplished by calculating heat transfer at the reported onset of boiling (i.e., $0.37 \mathrm{MW} / \mathrm{m}^{2} @ \Delta T=7{ }^{\circ} \mathrm{C}$ in Figure 23) and then linearly ramping this result to zero as the $\Delta T$ goes to zero. Calculating lower head response beyond the transition boiling region was not included and was not necessary because vessel failure is unavoidable after the $\mathrm{CHF}$ is reached. This occurs because bubble sizes increase as heat transfer increases until the $\mathrm{CHF}$ is reached. At that point, bubbles are large enough to begin blanketing the vessel, 


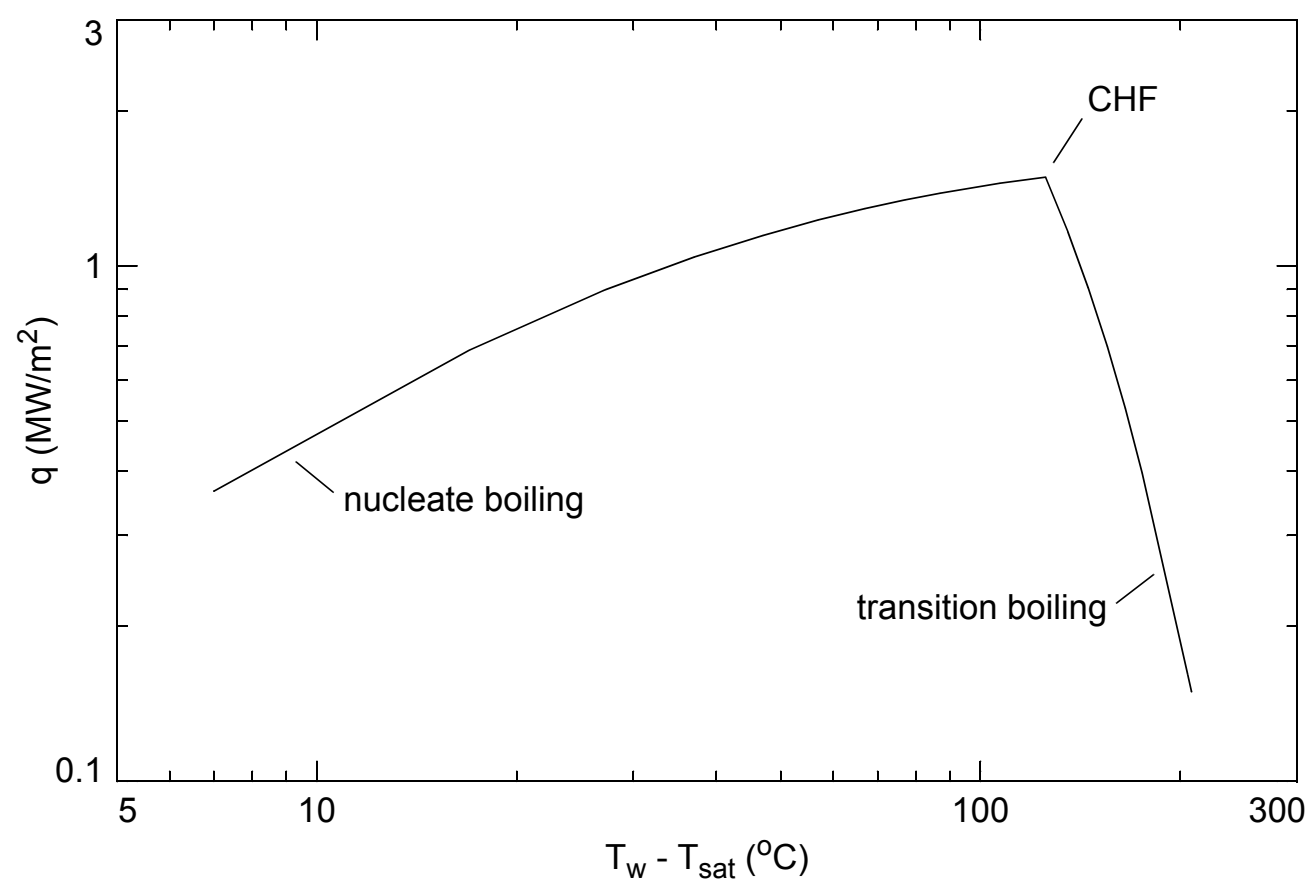

Figure 23. Curves for saturated boiling at atmospheric pressure from a vessel with a microporous coating at $0.98 \mathrm{rad}\left(56^{\circ}\right)$.

ultimately blocking water contact with the wall and limiting heat flow to that transferred across the steam film. The effect can be seen in Figure 23 where heat transfer actually drops as vessel wall temperatures increase beyond the CHF. The result is an unavoidable spiral of ever decreasing heat transfer and ever increasing wall temperatures. Burnout in the wall will eventually develop somewhere in a film boiling regime in such a case.

For each angular position of interest on the reactor vessel lower head, implementation of the code modifications just described included time-dependent logic to

- calculate the corresponding angle $(\theta)$,

- calculate the water saturation temperature consistent with the total pressure,

- determine the appropriate vessel configuration (plain, coated, insulated, or coated/insulated),

- calculate the corresponding CHF accounting for subcooling effects,

- use the current wall superheat $(\Delta T)$ to determine the appropriate heat transfer regime (pre-nucleate boiling, nucleate boiling, or transition boiling) based on data reported for the nearest angle,

- calculate heat transfer based on the current wall superheat and the curve associated with the appropriate heat transfer regime (which may require linear extrapolation from heat transfer at the onset of nucleate boiling to zero if the pre-nucleate boiling regime is applicable),

- scale heat transfer based on the calculated CHF,

- repeat the foregoing logic until the lower head temperature solution converges, and

- then repeat the foregoing logic to advance the simulation time. 


\subsection{Models and Input Assumptions}

All calculations were based on a simplified SCDAP/RELAP5-3D ${ }^{\complement}$ model of the lower head thermalhydraulics and a more detailed COUPLE model of the lower head structure. The SCDAP/RELAP5-3D ${ }^{\circledR}$ model, as shown in Figure 24, is consistent with the full plant model of the APR1400. However, the simplified version included time-dependent volumes to represent the core, downcomer, and the reactor cavity. Initial and boundary conditions were taken from results generated in Task 1 of this project.

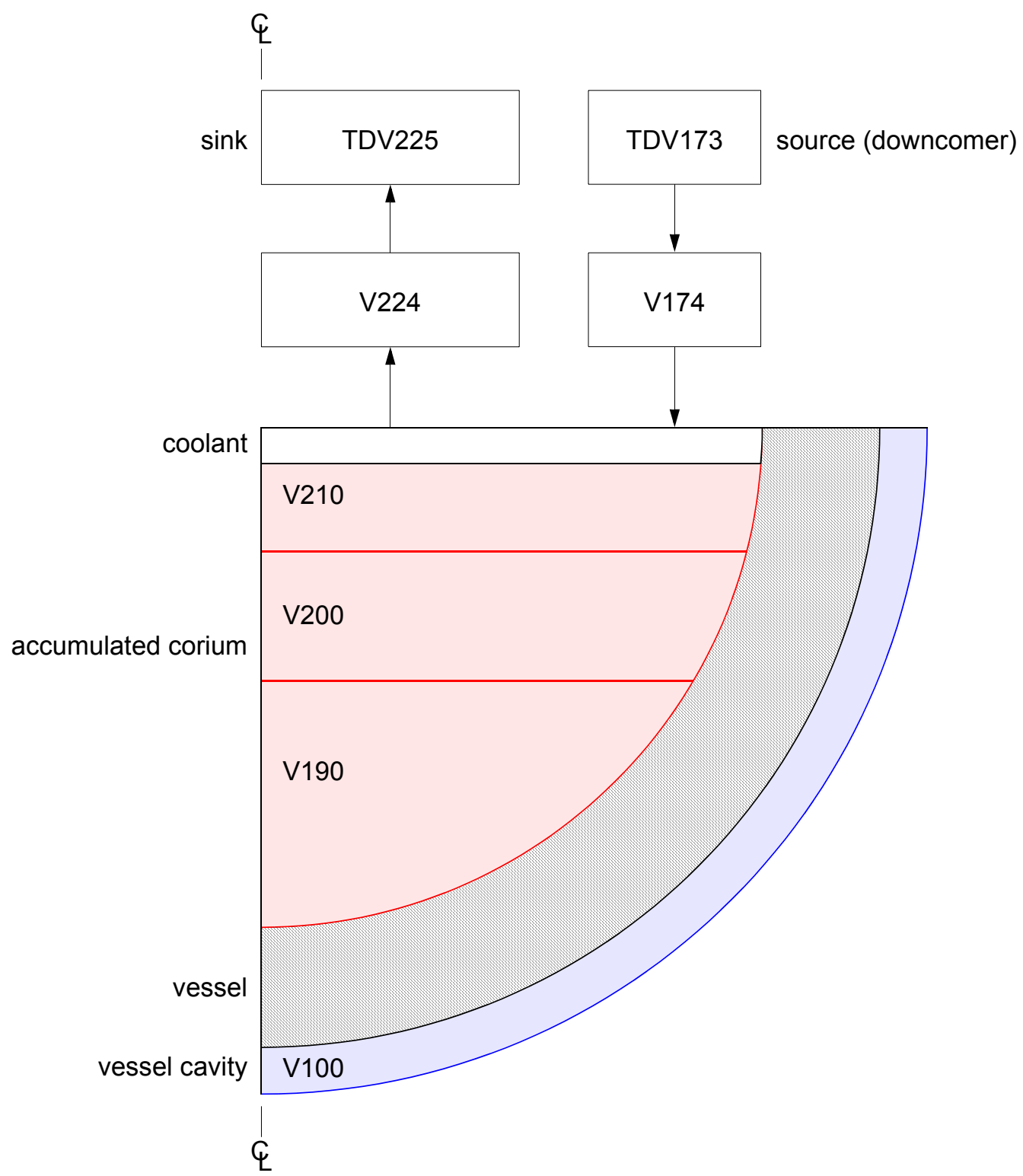

Figure 24. Simplified SCDAP/RELAP5-3D ${ }^{\complement}$ model of the APR1400 lower head thermal-hydraulics.

The lower head structure was modeled using the two-dimensional finite-element mesh shown in Figure 25. The mesh, which overlies three lower head hydrodynamic volumes (numbered V190, V200, 
and V210), includes a total of 588 nodes and 540 elements. Any transition from the lower head hemispherical geometry to the cylindrical vessel geometry was ignored due to the lack of specific information. Provisions were included, however, to allow simulation of a contact resistance between a solidified corium crust and the vessel wall, if a crust is predicted to form. Code modifications to simulate ERVC enhancements were applied to the exterior surfaces of this model.

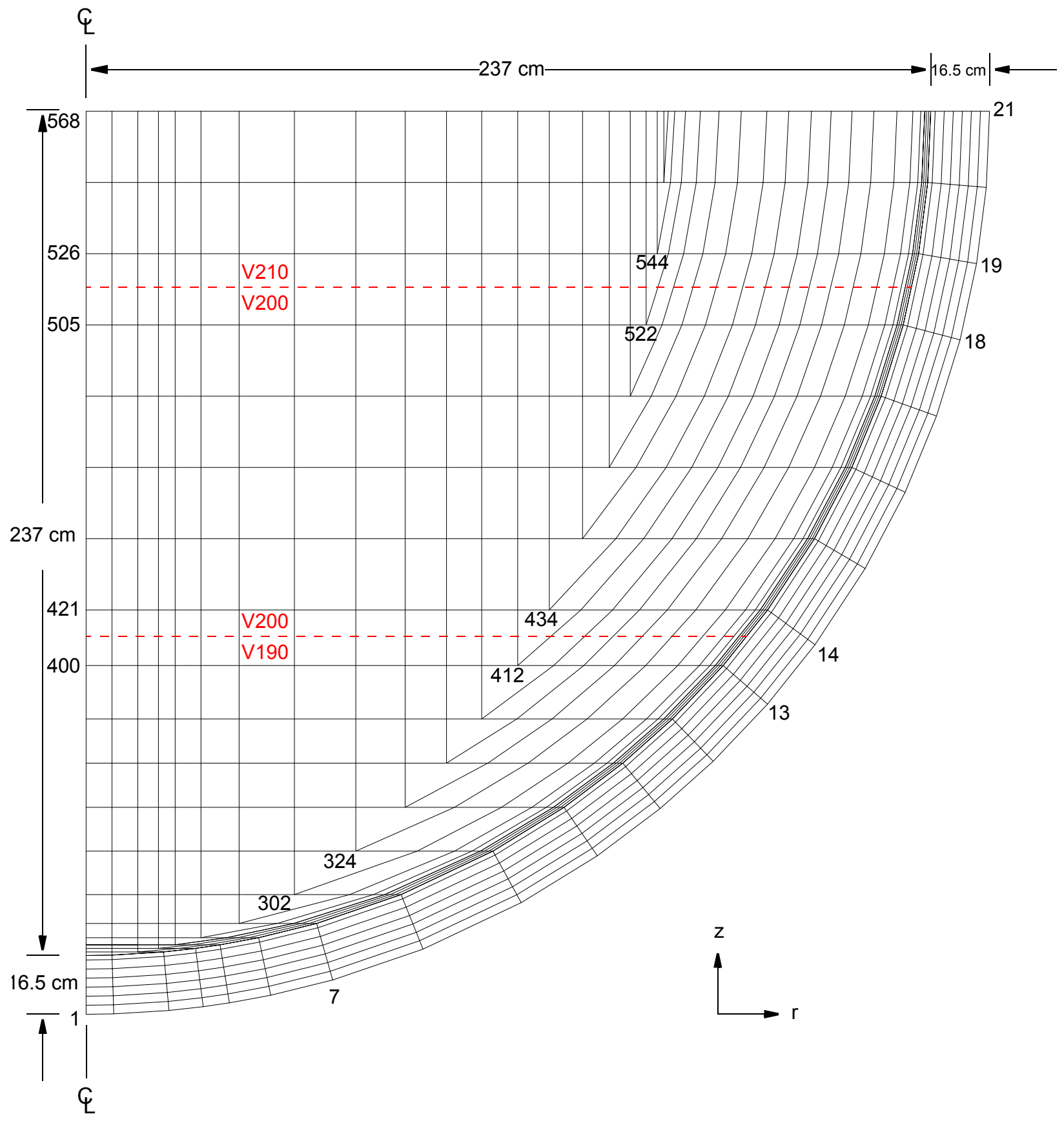

Figure 25. COUPLE two-dimensional finite-element mesh representing the lower head of the APR1400 reactor vessel (with selected node numbers). 
All calculations were completed using relocation conditions associated with a large loss-of-coolant accident (LOCA) in the APR1400. Those conditions, which are summarized as LOCA-1 in Table 3, were selected because they include the highest power density and the highest relocation temperature of all transients considered (under Task 1 of this project). In addition, the relocation conditions include a relatively large relocation mass and a relatively high decay power level. This is expected to result in a near limiting set of conditions relative to the severity of lower head thermal attack. (It should be noted that the probability of such conditions may be quite low. ${ }^{7}$ ) The actual relocation history associated with this transient and used in the calculations is provided in Table 4.

Table 3. Late-phase melt conditions for APR1400 transients.

\begin{tabular}{|c|c|c|c|c|c|c|c|c|c|c|}
\hline \multirow[b]{2}{*}{ Transient } & \multirow[b]{2}{*}{$\begin{array}{l}\text { Time of } \\
\text { Relocation } \\
\text { (s) }\end{array}$} & \multicolumn{4}{|c|}{ Relocated Constituents (kg) } & \multicolumn{5}{|c|}{ Corium Characteristics at Time of Relocation } \\
\hline & & $\mathrm{UO}_{2}$ & $\mathrm{ZrO}_{2}$ & $\mathrm{Zr}$ & Total & $\begin{array}{c}\text { Depth } \\
\text { (m) }\end{array}$ & $\begin{array}{c}\text { Temperature } \\
(\mathrm{K})\end{array}$ & $\begin{array}{l}\text { Decay } \\
\text { Power } \\
\text { (MW) }\end{array}$ & $\begin{array}{c}\text { Power } \\
\text { Density } \\
\left(\mathrm{MW} / \mathrm{m}^{3}\right)\end{array}$ & $\begin{array}{c}\text { Est Average } \\
\text { Vessel Heat Flux } \\
\left(\mathrm{MW} / \mathrm{m}^{2}\right)^{\mathrm{a}}\end{array}$ \\
\hline SBO-1 & 11,100 & 111,000 & 24,200 & 6,440 & $145,000^{\mathrm{b}}$ & 1.86 & 3,300 & 47.4 & 2.49 & 0.147 to 1.32 \\
\hline SBO-2 & 8,630 & 99,600 & 18,500 & 6,940 & $125,000^{\mathrm{c}}$ & 1.57 & 3,010 & 47.2 & 3.28 & 0.170 to 1.53 \\
\hline SBO-3 & 10,600 & 111,000 & 21,200 & 8,300 & $144,000^{\mathrm{d}}$ & 1.86 & 3,390 & 52.0 & 2.72 & 0.161 to 1.45 \\
\hline LOCA-1 & 4,990 & 108,000 & 5,180 & 3,520 & $119,000^{\mathrm{e}}$ & 1.60 & 3,460 & 51.2 & 3.48 & 0.182 to 1.64 \\
\hline
\end{tabular}

a. Assuming a hemispherical configuration, without sensible heat effects, for quasi-steady conditions, with estimated limits of heat loss from the upper corium surface (at 10 and $90 \%$ of the total decay heat level).

b. Includes $110 \mathrm{~kg} \mathrm{Zr}, 2,270 \mathrm{~kg}$ of control rod absorber material, and $76 \mathrm{~kg}$ of stainless steel from earlier relocations.

c. Includes $261 \mathrm{~kg}$ of control rod absorber material and $12 \mathrm{~kg}$ of stainless steel from earlier relocations.

d. Includes $250 \mathrm{~kg} \mathrm{Zr}, 2,350 \mathrm{~kg}$ of control rod absorber material, and $180 \mathrm{~kg}$ of stainless steel from earlier relocations.

e. Includes $276 \mathrm{~kg} \mathrm{Zr}, 2,350 \mathrm{~kg}$ of control rod absorber material, and $95 \mathrm{~kg}$ of stainless steel from earlier relocations.

Table 4. Relocation history for the APR 1400 LOCA-1 transient.

\begin{tabular}{c|c|c|c|c|c|c|c}
\hline \multirow{2}{*}{ Time (s) } & \multicolumn{5}{|c|}{ Relocated Mass (kg) } & \multicolumn{2}{c}{ Relocated Material } \\
\cline { 2 - 7 } & $\mathrm{UO}_{2}$ & $\mathrm{ZrO}_{2}$ & $\mathrm{Zr}$ & Steel & Absorber & Temperature (K) & Decay Power (MW) \\
\hline 3280 & & & & & 157 & 1480 & 1100 \\
3620 & & & & & 2033 & 1620 & \\
3700 & & & 93 & 32 & & 1560 & 1420 \\
4820 & & & 38 & 13 & 80 & 3460 & 51.2 \\
4990 & & & 145 & 50 & 80 & & \multicolumn{2}{c}{ Relocated Mass Totals (kg) } \\
\hline
\end{tabular}


The calculations were also completed assuming steady-state natural convection within molten regions of the (volumetrically-heated) corium pool. This is a somewhat conservative assumption because transient natural convection is lower than steady-state natural convection and because a finite period of time (which was ignored) is required before the transition from transient to steady-state convection will occur. The corium pool was assumed to be homogeneously mixed. This assumption was adopted out of consideration for time and budget constraints and the fact that sensitivities relative to segregation of the melt were treated in VESTA calculations. (SCDAP/RELAP5-3D ${ }^{\complement}$, however, does have provisions for modeling melt segregation.)

Conditions in the reactor vessel cavity were modeled as constants (although the code is capable of simulating any time-dependency of interest). A constant reactor cavity water depth of $7.1 \mathrm{~m}$ was included based on guidance from Korean collaborators. This should result in a flooding level near the elevation of the reactor hot legs. The cavity water was assumed to remain saturated at atmospheric pressure throughout the calculations.

\subsection{Results}

Models and input assumptions just described (in Section 3.3) were used in conjunction with code modifications outlined in Section 3.2 to evaluate the effects of ERVC enhancements on the potential for IVR in APR1400. During this process, however, a code deficiency was discovered. This deficiency arises from the fact that the code was never designed to explicitly simulate any significant melting of the reactor vessel. Given this discovery, it seems appropriate to distinguish between code deficiencies and code errors. After this distinction is made, specific information regarding the nature of the deficiency and steps to remedy the problem will be discussed.

Errors occur if/when the code fails to perform as designed. On the other hand, deficiencies can surface if/when the code is used beyond its design limits. In this particular application, significant melting of the reactor vessel occurs. As such, the results lie in a realm beyond the design of the code. Hence, the problem with the code is best described as a deficiency, not an error.

The deficiency basically arises from details associated with modeling heat transfer from the molten corium to the reactor vessel. As the code currently exists, there are two possibilities associated with this heat transfer path: one where some amount of corium has solidified to form a crust adjacent to the inner surface of the vessel wall and one without a solidified crust.

The most common situation, at least in previous applications, is the case where heat transfer from the molten corium is high enough, relative to the corium decay power, to lead to the formation of a solidified corium crust. In this case, heat flows by convection to the inner most crust surface, by conduction through the crust, through a crust-to-vessel contact resistance, by conduction through the vessel, and finally, through convection to the cavity water. Under these conditions, the reactor vessel is protected from melting by the presence of the solidified crust. The other possibility for heat transfer from the molten corium to the reactor vessel arises when the heat transfer from the corium is low, relative to the decay power, so that temperatures near the vessel remain high and a solidified corium crust adjacent to the reactor vessel does not form. Under these conditions, the vessel is more directly exposed to molten pool temperatures, which is the situation encountered in this analysis. This result appears to be a direct consequence of modeling high power reactors with decay heat levels above those previously considered. 
The code automatically simulates corium crust growth and shrinkage throughout all calculations. This is accomplished by comparing heat added to the crust from the molten pool side to the heat removed from the crust on the vessel side. If more heat is added than removed, the crust must shrink consistent with the conservation of energy. If/when the crust completely disappears, the code currently uses an arbitrary fixed heat transfer coefficient of $10,000 \mathrm{~W} / \mathrm{m}^{2}-\mathrm{K}$ to approximate direct molten corium-to-vessel heat transfer. This fixed coefficient was selected partly as a means to promote rapid crust solidification, as expected during the relocation process while the vessel is relatively cold. Thereafter, it was implicitly assumed that heat transfer through the vessel will be sufficient, relative to the decay power, to maintain some crust thickness. Selection of this fixed coefficient was also influenced by the fact that experimental data for this heat transfer situation does not exist.

When the fixed coefficient (of $10,000 \mathrm{~W} / \mathrm{m}^{2}-\mathrm{K}$ ) is applied in these calculations, very rapid vessel melting occurs. The process is so rapid that melt-through is near complete before vessel surface temperatures have time to increase. With vessel surface temperatures relatively low, heat rejection to water in the cavity remains relatively low, which tends to further promote the melting process. Given those results, comparisons of the heat flux from the molten corium pool with the heat flux to the vessel wall were needed.

Heat flux from the side of the molten corium pool was calculated using correlations contained in the code for steady state natural convection in a volumetrically heated pool given by ${ }^{18,19}$

$h=0.54\left(\frac{k}{R}\right) R a^{0.18} f(\theta)$

where

$h=$ local heat transfer coefficient,

$k=$ thermal conductivity of the melt in the boundary layer adjacent to the interface,

$R \quad=$ effective radius of the molten region (in a hemispherical geometry),

$R a=\frac{g \beta \dot{Q} R^{5}}{\alpha v k}=$ Rayleigh number associated with the molten pool,

$g \quad=$ gravitational constant,

$\beta=$ coefficient of volumetric expansion,

$\dot{Q}=$ volumetric heat generation rate,

$\alpha=$ thermal diffusivity,

$v \quad=$ kinematic viscosity of the molten materials, and

$f(\theta)=$ the relationship between local and mean heat transfer reflected in Figure 26.

After a limited number of comparisons, it became clear that code use of the fixed coefficient of $10,000 \mathrm{~W} / \mathrm{m}^{2}-\mathrm{K}$ results in heat flow to the inner surface of the vessel wall well above that predicted by Eq. (3). In fact, discrepancies ranged from $\sim 100$ to $\sim 300 \%$ for positions and points in time that were sampled. In other words, the use of $10,000 \mathrm{~W} / \mathrm{m}^{2}-\mathrm{K}$ can yield vessel heat loads that far exceed the decay power generated in the molten pool. 


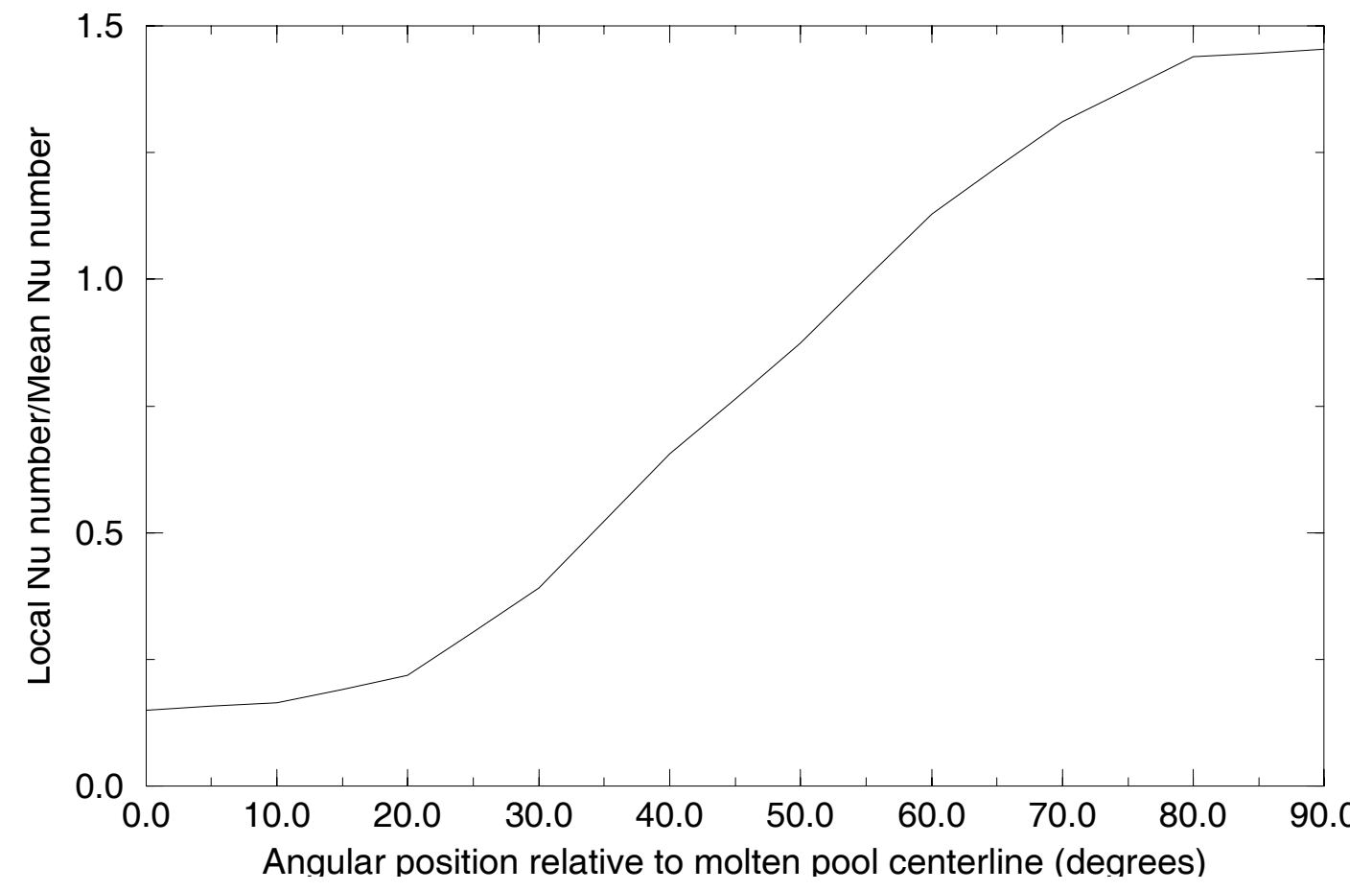

Figure 26. Nusselt number ratio as a function of the angle from molten pool centerline.

Time and budget constraints in this project did not allow resolution of this code deficiency. However, the following represents some of the modifications needed to add code capabilities to appropriately account for the absence of a solidified corium crust and melting of the reactor vessel wall.

- The arbitrary fixed heat transfer coefficient of $10,000 \mathrm{~W} / \mathrm{m}^{2}-\mathrm{K}$ from the molten pool to the inner surface of the vessel wall should be replaced with time-dependent values calculated to yield heat flow equal to that associated with natural convection from the volumetrically heated molten pool. This approach is expected to result in an upper bound on heat flow to the wall because any convective film drop is ignored. This could be refined if/when experimental data for the specific convective process become available.

- Vessel melting, including the latent heat of fusion associated with melting the reactor vessel wall, should be added to SCDAP/RELAP5-3D ${ }^{\complement}$.

- The composition of the molten pool should be modified as a function of time to account for the progression of vessel steel melting.

- Thermal properties of the molten pool should replace the thermal properties of steel in those regions where the reactor vessel has melted.

Although these modifications could not be implemented during this project, some insights may be gained by reviewing results from a limited number of calculations that were completed. In these calculations, the molten pool-to-vessel heat transfer coefficient was reduced from 10,000 to $600 \mathrm{~W} / \mathrm{m}^{2}-\mathrm{K}$. This reduction was specifically selected so complete melt through would not occur in vessels with microporous coatings. The basis for this selection was not entirely arbitrary; instead, it was noted that 
- heat transfer coefficients that would be needed to yield heat flow equal to that associated with natural convection from the volumetrically heated molten pool were much closer to 600 than $10,000 \mathrm{~W} / \mathrm{m}^{2}-\mathrm{K}$,

- the convective heat flux from the molten pool predicted using Eq. (3) is less than the CHF (as long as molten pool temperatures remain reasonable),

- scoping calculations indicate that the CHF from vessels with microporous coatings should be sufficient to reject the total decay power (of 51.2 MW), and

- VESTA results (Section 2.6) indicate that vessels with microporous coatings will not completely melt through.

The ratio of vessel surface heat flux to the CHF for vessels with microporous coatings at $9000 \mathrm{~s}$ $(\sim 1.1 \mathrm{hr}$ after molten fuel relocation) is shown in Figure 27. These results indicate that the selected molten corium-to-vessel heat transfer coefficient of $600 \mathrm{~W} / \mathrm{m}^{2}-\mathrm{K}$ can lead to large vessel surface heat fluxes, with some closely approaching the CHF at specific angular positions. As desired, however, the selected coefficient was just adequate to prevent complete melt through of coated vessels. The asymptotic behavior of vessel wall temperatures shown in Figure 28 would indicate that wall melting has stabilized by $\sim 8000 \mathrm{~s}$ and that further increases in heat flux ratios (with additional time) will be small. Figure 28 also indicates the approach to a quasi-stable vessel thickness of $\sim 5 \mathrm{~cm}$. Similar vessel wall thinning due to melting extended over the region from Nodes 8 to 16 (see node locations in Figure 25). Results shown in Figure 29 support this observation.

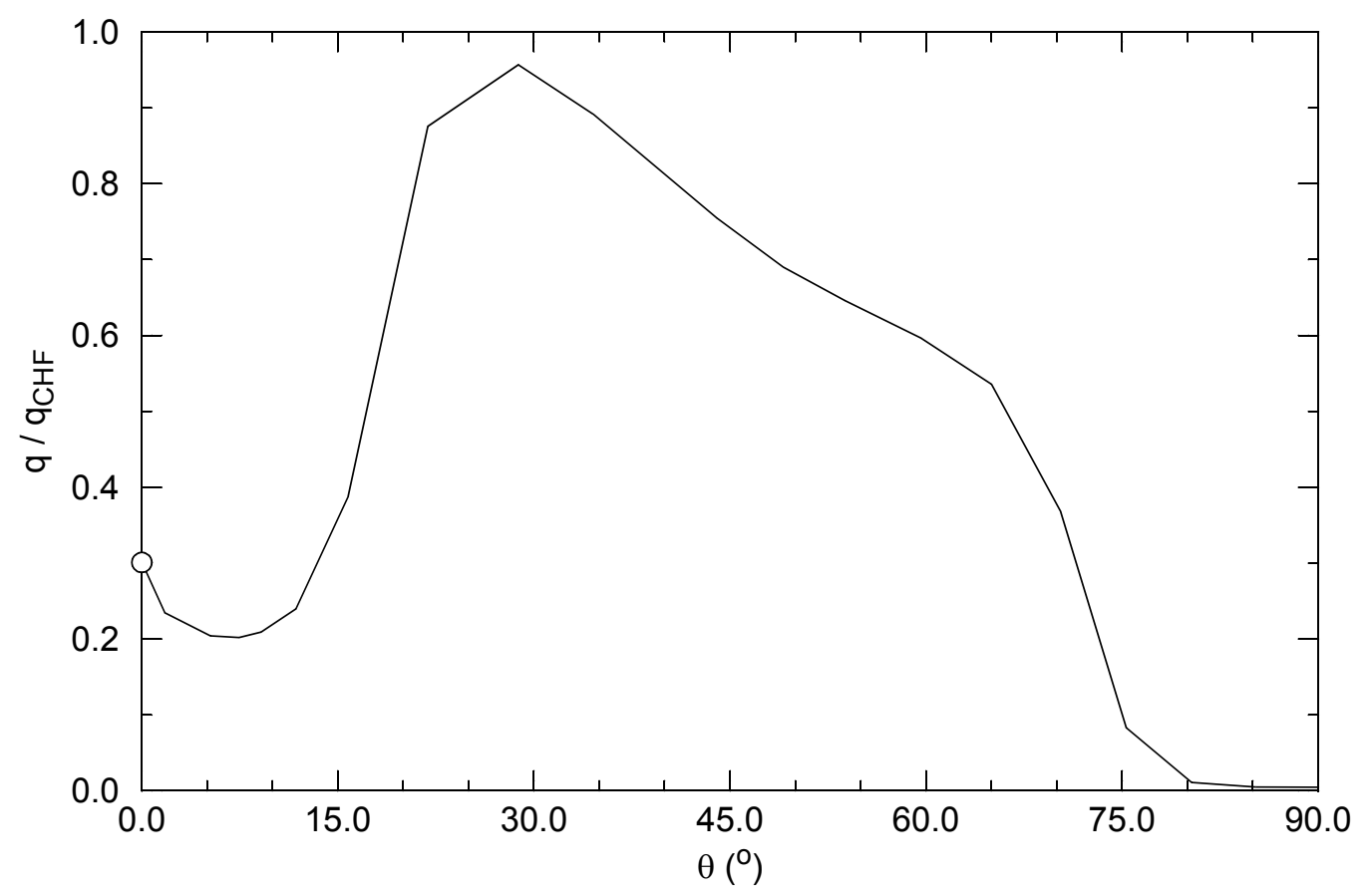

Figure 27. The ratio of vessel surface heat flux to the CHF for a vessel with a microporous coating at $9000 \mathrm{~s}$. 


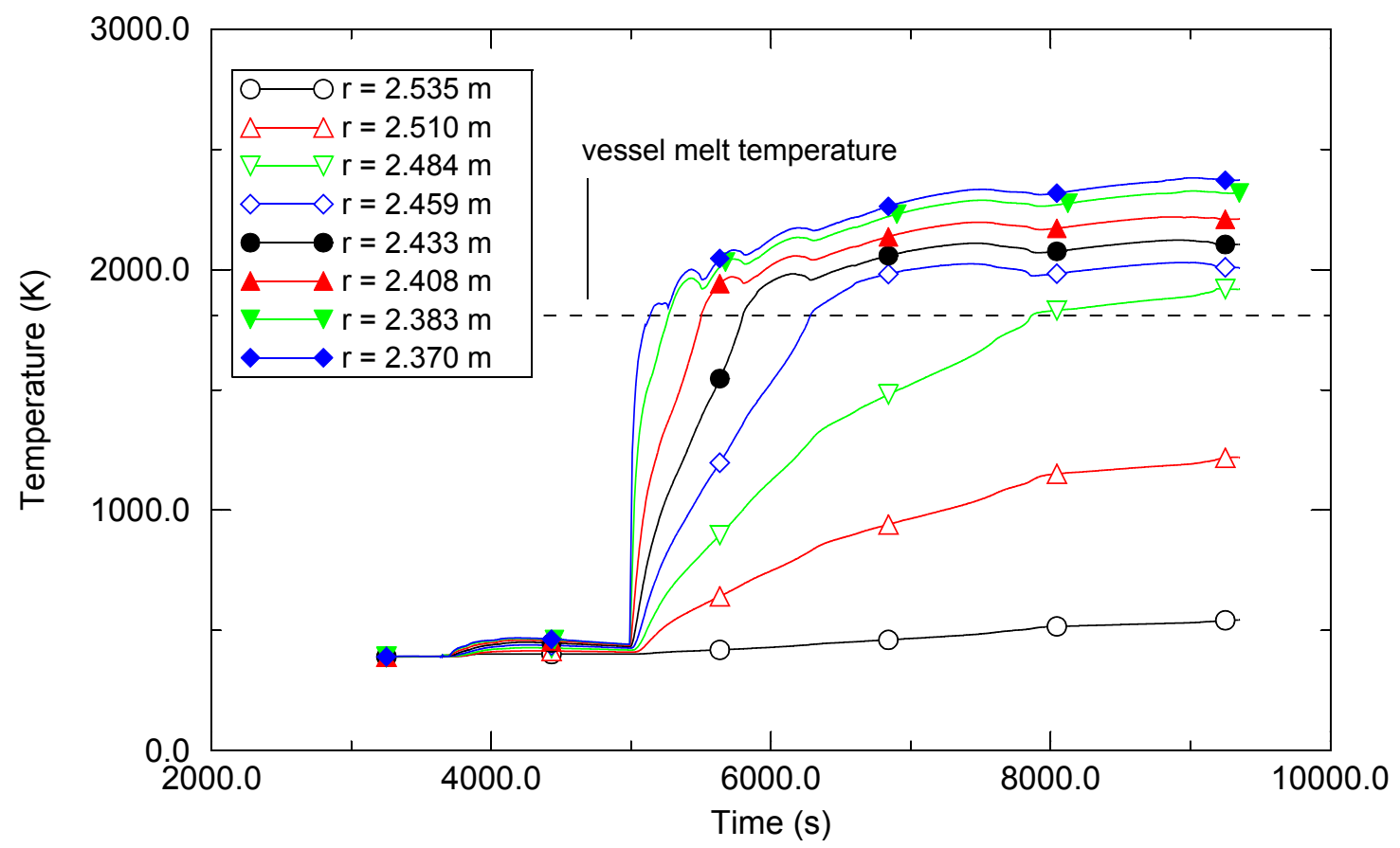

Figure 28. Through-wall temperature profile (from Node 9 in Figure 25) for a vessel with a microporous coating.

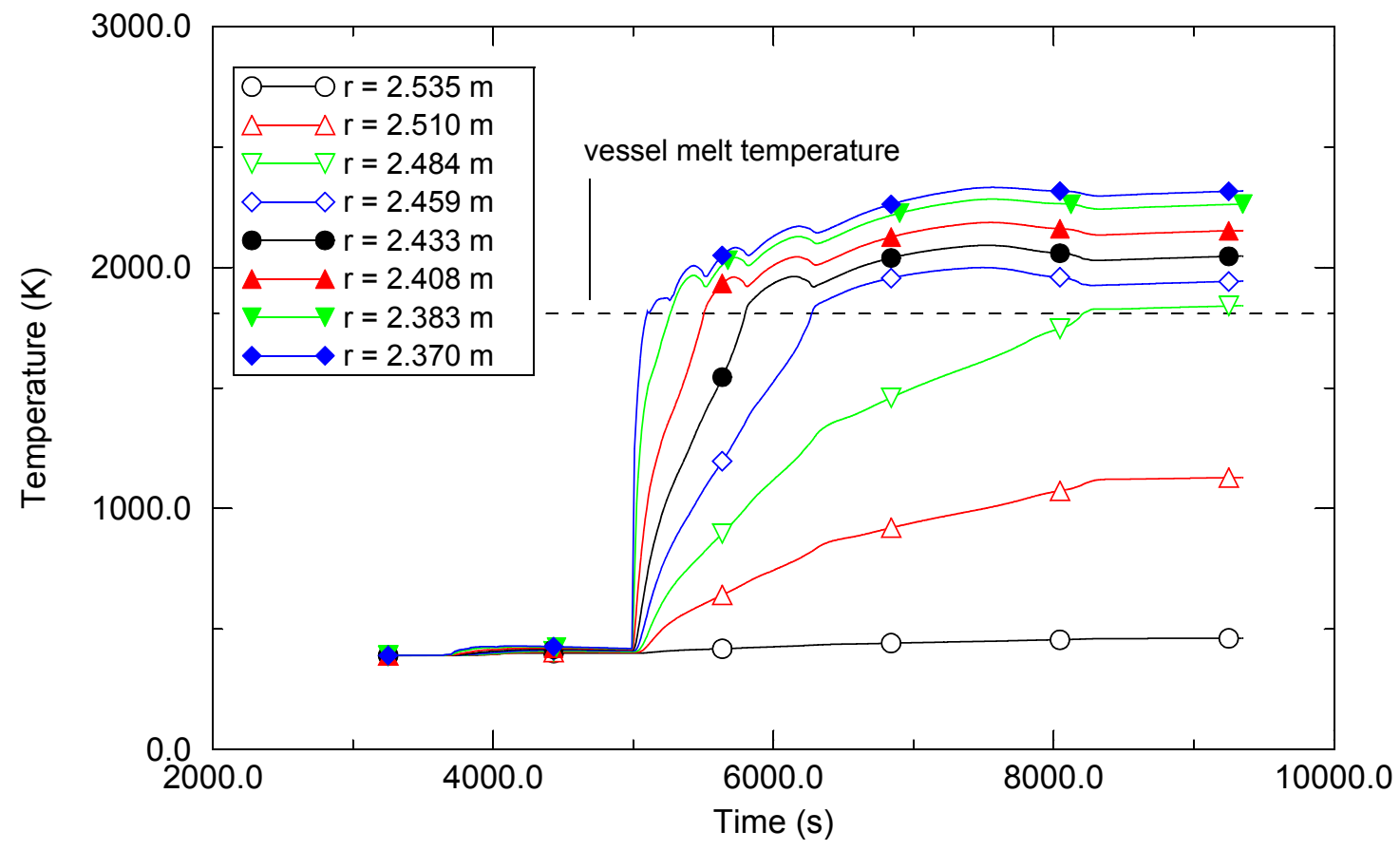

Figure 29. Through-wall temperature profile (from Node 12 in Figure 25) for a vessel with a microporous coating. 
A limitation of using any fixed molten corium-to-vessel heat transfer coefficient is that a constant can never correctly account for heat transfer variations that can develop with time. In calculations for coated vessels, this limitation becomes most apparent with respect to ever-increasing molten pool temperatures. As pool temperatures increase, heat flux from the corium to the vessel increases (consistent with Eq. (3). Results shown in Figure 30 reflect this trend. Considerable improvement in the simulation would be expected if time-dependent coefficients were calculated to balance heat flow to the vessel with heat flow from the corium. Assuming the total decay power does not exceed CHF limits, one would expect corium temperatures to stabilize and then gradually decrease under these conditions. Results shown in Figure 31 were generated at a time before most of the corium pool temperature increase. As such, these results may be more representative of heat flux ratios that may be achieved with appropriate code modifications.

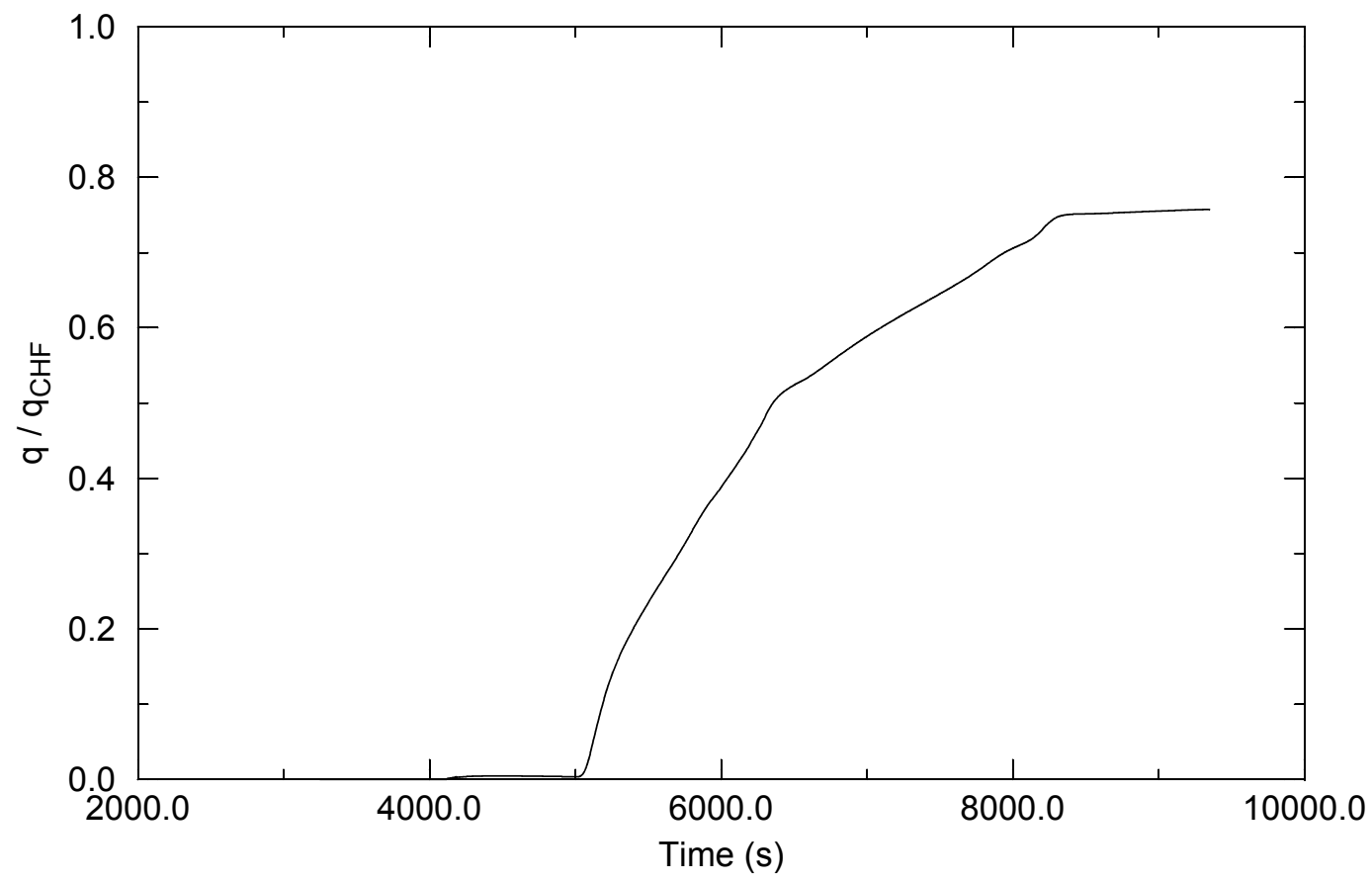

Figure 30. The ratio of vessel surface heat flux to the CHF for Node 12 on a vessel with a microporous coating (see Figure 25 for node position).

Calculations with a fixed corium-to-vessel heat transfer coefficient of $600 \mathrm{~W} / \mathrm{m}^{2}-\mathrm{K}$ were also completed for plain vessels. Although use of a fixed coefficient has limitations, this at least provides a common basis for comparison with coated vessels results.

Results for plain vessels indicate complete melt through over a region bounded by Nodes 7 and 12 by $9000 \mathrm{~s}(\sim 1.1 \mathrm{hr}$ after molten fuel relocation). Figures 32 and 33 are representative of the vessel thermal response in the failed regions. Based on the results shown, further melting is probable given that temperatures are continuing to rise. Failure of plain vessels for the modeled conditions is not unexpected because

- the convective heat flux from the molten pool predicted using Eq. (3) is greater than the CHF (even at moderate molten pool temperatures), 


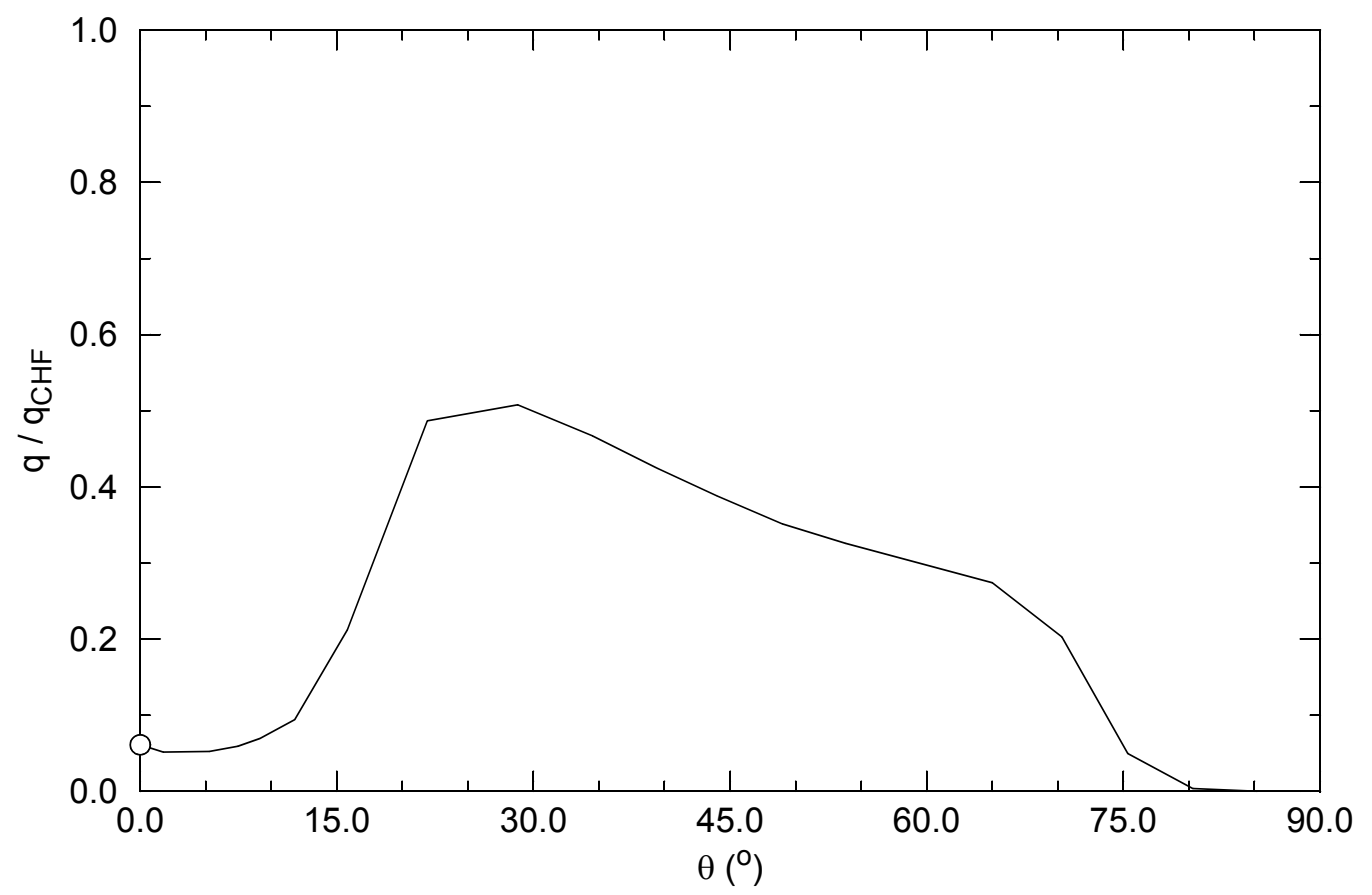

Figure 31. The ratio of vessel surface heat flux to the CHF for a vessel with a microporous coating at $6000 \mathrm{~s}$.

- scoping calculations indicate that the CHF from plain vessels is not sufficient to reject the total decay power (of 51.2 MW), and

- VESTA results (Section 2.6) indicate that plain vessels will melt through.

Complete melt through in the plain vessel calculation stands in contrast to the results for the coated vessel where wall thinning was ultimately controlled by ERVC. Although the absolute value of the results can be questioned (because a fixed corium-to-vessel coefficient was used), the results clearly indicate significant IVR benefits are achievable through the use of microporous coatings. Given time and budget constraints and the limitations associated with the identified code deficiency, a decision was made to defer calculations for the remaining ERVC enhancement options (i.e., insulated and coated/insulated vessels). Results that were calculated and the information provided in Figure 4, however, is sufficient to indicate that IVR benefits can be extended by exercising insulated and coated/insulated ERVC enhancement options. SCDAP/RELAP5-3D ${ }^{\complement}$ modifications are needed before these improvements can be quantified. The effort to complete the code modifications certainly appears justified given the potential benefits for validating IVR in high power reactors.

\subsection{Summary}

SCDAP/RELAP5-3D ${ }^{\complement}$ was modified to allow simulation of ERVC from reactor vessels with microporous coatings, optimized insulation, and the combination of coatings and insulation based on PSU experimental results. Modifications were also added to simulate ERVC from plain vessels as a reference for gauging any potential IVR improvements that may be derived from implementing any of the ERVC 


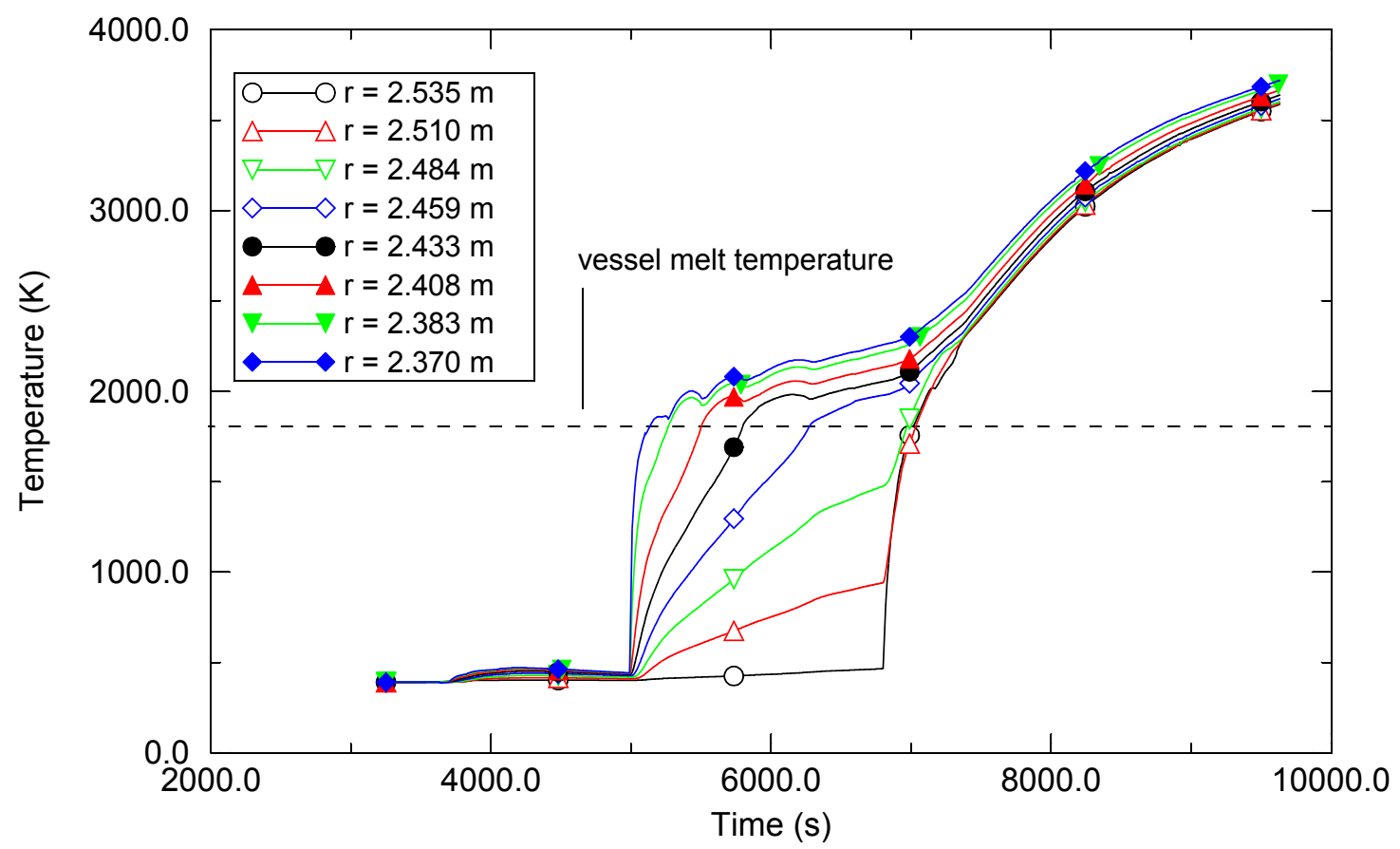

Figure 32. Through-wall temperature profile (from Node 9 in Figure 25) for a plain vessel.

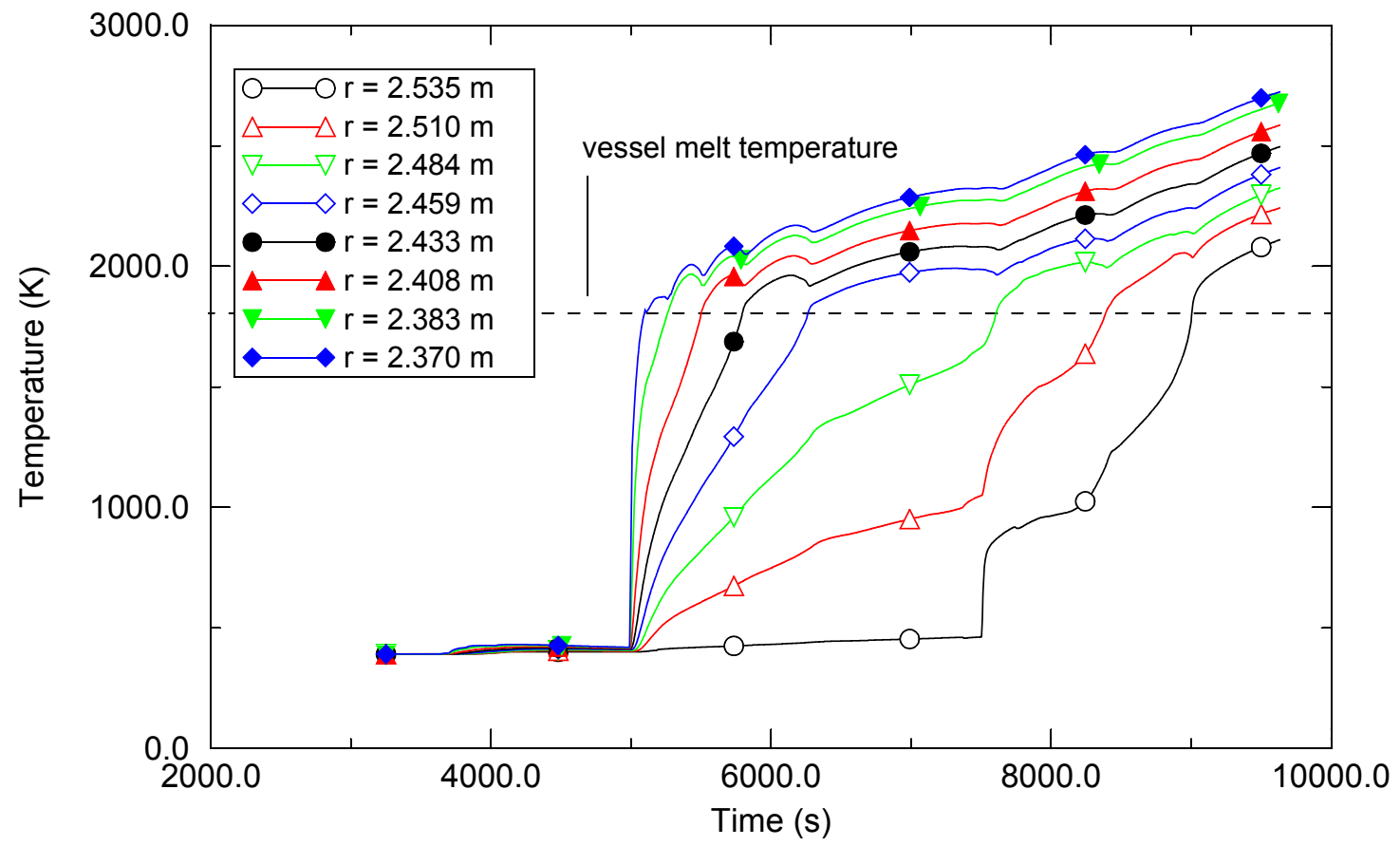

Figure 33. Through-wall temperature profile (from Node 12 in Figure 25) for a plain vessel. 
enhancements. Models of the Korean APR1400 lower head were developed to allow evaluation the potential for IVR in high power reactors.

Calculations using the ERVC modifications and the APR1400 model led to discovery of a code deficiency. The deficiency is related to the fact that the code was not designed to simulate significant reactor vessel melting. The following recommendations were derived after evaluating this deficiency.

- The arbitrary fixed heat transfer coefficient of $10,000 \mathrm{~W} / \mathrm{m}^{2}-\mathrm{K}$ from the molten pool to the inner surface of the vessel wall should be replaced with time-dependent values calculated to yield heat flow equal to that associated with natural convection from the volumetrically heated molten pool.

- Vessel melting, including the latent heat of fusion associated with melting the reactor vessel wall, should be added to SCDAP/RELAP5-3D ${ }^{\complement}$.

- The composition of the molten pool should be modified as a function of time to account for the progression of vessel steel melting.

- Thermal properties of the molten pool should replace the thermal properties of steel in those regions where the reactor vessel has melted.

Because project time and budget constraints precluded resolution of the code deficiency, a limited number of calculations were completed using a more reasonable molten pool-to-vessel coefficient of $600 \mathrm{~W} / \mathrm{m}^{2}-\mathrm{K}$. Results from these calculations indicate plain vessels will fail by melt through while coated vessel wall thinning will be limited (before vessel failure) by ERVC, given the same modeling assumptions and limitations. Although the absolute value of the results can be questioned (because a fixed coriumto-vessel coefficient was used), comparing plain and coated vessel results clearly indicates the use of microporous coatings has significant IVR benefits. Available information is sufficient to indicate that IVR benefits can be extended by exercising insulated and coated/insulated ERVC enhancement options. SCDAP/RELAP5-3D ${ }^{\odot}$ modifications are needed before these improvements can be quantified. The effort to complete the code modifications certainly appears justified given the potential benefits for validating IVR in high power reactors.

\section{Conclusions}

As a final task in this INERI, VESTA and SCDAP/RELAP5-3D ${ }^{\odot}$ codes were applied to assess the impact of proposed enhancements for IVR. Two debris endstate configurations were considered in the VESTA calculations: Configuration A, a stratified configuration with an upper metallic layer; and Configuration $\mathrm{C}$, a stratified configuration with a lower metallic layer.

VESTA results for base case conditions in a Configuration A endstate indicate:

- Peak heat fluxes and associated CHF ratios occur near the top of the ceramic pool beneath the metallic layer.

- A small, but non-zero, probability exists for the CHF ratio to exceed 1.0.

- An IVCC must prevent melt relocation for at least 4 hours in order to maintain vessel heat fluxes below the CHF.

- Either of the explored ERVC enhancements are sufficient to reduce vessel heat fluxes below the CHF. If one considers uncertainties, the additional reduction offered by an enhanced insulation design is required to maintain heat fluxes below the CHF. 
- An IVCC must decrease heat fluxes by at least a factor of 2 for this configuration.

- ERVC enhancements may decrease heat fluxes by factors of 2 to 5 .

VESTA results for base case conditions in a Configuration $\mathrm{C}$ endstate suggest:

- Higher heat fluxes occur at vessel locations adjacent to the ceramic layer.

- CHF ratios peak at two locations for this configuration: near the bottom center of the vessel and near the top of the ceramic pool.

- Ether an IVCC, which can prevent relocation onto the vessel for at least 4 hours) or a vessel with the combined ERVC enhancements considered in this study are sufficient to maintain vessel heat fluxes below the CHF.

VESTA results suggest that either of the proposed IVR enhancements can preclude vessel heat fluxes from exceeding the CHF. Depending upon the selected IVR enhancement and debris endstate configuration, IVR margins may increase by factors ranging from two to four. The benefit associated with each enhancement is dependent upon the debris endstate (a condition that is not possible to predict at this time).

SCDAP/RELAP5-3D ${ }^{\complement}$ was modified to allow simulation of ERVC from reactor vessels with microporous coatings, optimized insulation, and the combination of coatings and insulation based on PSU experimental results. Modifications were also added to simulate ERVC from plain vessels as a reference for gauging any potential IVR improvements that may be derived from implementing any of the ERVC enhancements. Models of the Korean APR1400 lower head were developed to allow evaluation the potential for IVR in high power reactors. This evaluation was needed because SCDAP/RELAP5-3D ${ }^{\odot}$ provides the only analytical approach for predicting certain critical aspects of APR1400 behavior, particularly the simulation of transients and the treatment of complex boundary conditions.

SCDAP/RELAP5-3D ${ }^{\complement}$ calculations using the ERVC modifications and the APR1400 model led to discovery of a code deficiency. The deficiency is related to the fact that the code was not designed to simulate significant reactor vessel melting. Because project time and budget constraints precluded resolution of the deficiency, a limited number of calculations were completed using a fixed molten pool-to-vessel coefficient of $600 \mathrm{~W} / \mathrm{m}^{2}-\mathrm{K}$. Results from these calculations indicate plain vessels will fail by melt through while coated vessel wall thinning will be limited (before vessel failure) by ERVC, given the same modeling assumptions and limitations. Although the absolute value of the results can be questioned (because a fixed coefficient was used), comparing plain and coated vessel results clearly indicates the use of microporous coatings has significant IVR benefits. Available information is sufficient to indicate that IVR benefits can be extended by exercising insulated and coated/insulated ERVC enhancement options. SCDAP/RELAP5-3D ${ }^{\complement}$ modifications are needed before these improvements can be quantified.

In completing this work, the following tasks were identified that could not be completed due to budget and schedule limitations:

- Additional VESTA sensitivity studies to assess the impact of several input parameters and associated uncertainty distributions that were based on the Reference 11 analyses.

- Additional debris endstate configurations. VESTA currently models 3 distinct endstate configurations and SCDAP/RELAP5-3D ${ }^{\complement}$ currently models 2 endstate configurations. However, budget and time constraints limited the number of configurations that could be completed for this INERI.

- A detailed SCDAP/RELAP5-3D ${ }^{\odot}$ calculation to determine IVCC failure time and associated decay heat at the time that melt relocates to the vessel lower head. This SCDAP/RELAP5-3D ${ }^{(}$ 
would incorporate narrow gap cooling correlations that were recently developed in this INERI. VESTA calculations would then be performed using the IVCC failure time and a decay heat that is revised to considered the IVCC failure time and any dilution due to the IVCC being subsumed in the relocated corium.

- Additional VESTA calculations using a version that is updated with newly obtained heat transfer correlations to simulate natural convection heat transfer in a volumetrically heated pool.

- The arbitrary fixed heat transfer coefficient of $10,000 \mathrm{~W} / \mathrm{m}^{2}-\mathrm{K}$ from the molten pool to the inner surface of the vessel wall currently in SCDAP/RELAP5-3D ${ }^{\odot}$ should be replaced with time-dependent values calculated to yield heat flow equal to that associated with natural convection from the volumetrically heated molten pool.

- Vessel melting, including the latent heat of fusion associated with melting the reactor vessel wall, should be added to SCDAP/RELAP5-3D ${ }^{\complement}$.

- The composition of the SCDAP/RELAP5-3D ${ }^{\odot}$ molten pool should be modified as a function of time to account for the progression of vessel steel melting.

- Thermal properties of the SCDAP/RELAP5-3D ${ }^{\complement}$ molten pool should replace the thermal properties of steel in those regions where the reactor vessel has melted.

Although the above tasks are not included in the workscope for this INERI, results from these tasks would provide additional insights about the benefits of proposed ERVC enhancements. The effort to complete these tasks certainly appears justified given the potential benefits of verifying IVR in high power reactors.

\section{References}

1. J. L. Rempe, K. Y. Suh, F.-B. Cheung, and S.-B. Kim, "In-Vessel Retention Strategy for High Power Reactors In-Vessel Retention Strategy for High Power Reactors - Final Report,” INEEL-EXT-???, December 2004.

2. J. Rempe, et al., "An Enhanced In-Vessel Core Catcher for Improving In-Vessel Retention Margins," Invited paper, Nuclear Technology NURETH10 Special Edition, to be published 2005.

3. Y. H. Kim, S. J. Kim, S. W. Noh, K. Y. Suh, J. L. Rempe, F. B. Cheung, S. B. Kim, "Visualization of Boiling Phenomena in Inclined Rectangular Gap, submitted to the International Journal of Multiphase Flow, April 2004.

4. M. B. Dizon, J. Yang, F. B. Cheung, J. L. Rempe, K. Y. Suh, and S. B. Kim, "Effects of Surface Coating on the Critical Heat Flux for Pool Boiling from a Downward Facing Surface, Journal of Enhanced Heat Transfer, Vol. 11, pp. 133-150, 2004.

5. F. B. Cheung, J. Yang, M. B. Dizon, J. L. Rempe, K. Y. Suh, and S. B. Kim, "Scaling of Downward Facing Boiling and Steam Venting in a Heated Hemispherical Annular Channel," International Journal of Transport Phenomena, Vol. 6, pp. 81-96, 2004.

6. J. L. Rempe, D. L. Knudson, K. G. Condie, K. Y. Suh, F. B. Cheung, and S. B. Kim, "Conceptual Design of an In-Vessel Core Catcher," Nuclear Engineering and Design, invited paper for ICONE11 Special Edition, 230 (2004) 311-235. 
7. D. L. Knudson, J. L. Rempe, K. G. Condie, K. Y. Suh, F. B. Cheung, and S. B. Kim, "Late-Phase Melt Conditions affecting the Potential for In-Vessel Retention in High Power Reactors," Nuclear Engineering and Design, invited paper for ICONE11 Special Edition, 230 (2004), 133-150.

8. J. L. Rempe, D. L. Knudson, K. G. Condie, K. Y. Suh, F. B. Cheung, and S. B. Kim, "Corium Retention for High Power Reactors by an In-Vessel Core Catcher in Combination with External Reactor Vessel Cooling," Nuclear Engineering and Design, invited paper for ICONE11 Special Edition, 230 (2004), 293-309.

9. C. S. Kim, K. Y. Suh, J. L. Rempe, F. B. Cheung, S. B. Kim, "Effect of Interfacial Wavy Motion on Film Boiling Heat Transfer from Isothermal Downward-facing Hemispheres," submitted to Nuclear Engineering and Design, August 2004.

10. F. B. Cheung, J. Yang, and M. B. Dizon, "SBLB Results for Task 3 on External Reactor Vessel Cooling (ERVC) Boiling Data and CHF Enhancement Correlations," PSU Letter Report \#PSU/MNE-2004INEEL-2, September 30, 2004.

11. J. L. Rempe, et al., Potential for In-Vessel Retention through Ex-vessel Flooding, INEEL External Report INEEL/EXT-97-00779, December 1997. Document submitted to ACRS as part of AP600 design certification review.

12. J. L. Rempe, et. al, "Impact of Uncertainties on the Response of an Externally Flooded Vessel," published in the transactions from the Fourth International Conference on Probabilistic Safety Assessment and Management (PSAM-4), September 13-18, 1998, New York, NY.

13. J. L. Rempe, et al, "Evaluation of In-Vessel Retention through Ex-vessel Flooding," Transactions from the Sixth International Conference on Nuclear Engineering (ICONE-6), May 1998, San Diego, CA.

14. J. L. Rempe, et al., "Potential for In-Vessel Retention through Ex-vessel Flooding," paper published in transactions of OECD/ CSNI Workshop on In-Vessel Debris Retention and Coolability, March 3-6, 1998, Garching, Germany.

15. T. G. Theofanous et al., In-Vessel Coolability and Retention of a Core Melt, DOE/ID-10460, Revised October 1996.

16. OECD MASCA Project website: www.nsi.kiae.ru.

17. The SCDAP/RELAP5-3D ${ }^{\odot}$ Development Team, SCDAP/RELAP5-3D ${ }^{\complement}$ Code Manuals, INEEL/EXT02/00589, Idaho National Engineering and Environmental Laboratory, May 2002.

18. F. Mayinger et al., Examination of Thermal-Hydraulic Processes and Heat Transfer in a Core Melt, BMFT RS 48/1, Institute for Verfahrenstechnik der T. U. Hanover, 1976.

19. M. Jahn and H. H. Reineke, "Free Convection Heat Transfer with Internal Heat Source, Calculations and Measurements", Proceedings of the International Meeting on Thermal Nuclear Reactor Safety, NUREG/CR-0027, February 1983. 